\title{
INFLUENCE OF STELLAR MULTIPLICITY ON PLANET FORMATION. II. PLANETS ARE LESS COMMON IN MULTIPLE-STAR SYSTEMS WITH SEPARATIONS SMALLER THAN $1500 \mathrm{AU}$
}

\author{
Ji WANG ${ }^{1}$, Debra A. Fischer ${ }^{1}$, Ji-Wei XiE ${ }^{2}$, AND David R. Ciardi ${ }^{3}$ \\ ${ }^{1}$ Department of Astronomy, Yale University, New Haven, CT 06511, USA; ji.wang@yale.edu \\ ${ }^{2}$ Department of Astronomy \& Key Laboratory of Modern Astronomy and Astrophysics \\ in Ministry of Education, Nanjing University, Nanjing 210093, China \\ ${ }^{3}$ NASA Exoplanet Science Institute, Caltech, MS 100-22, 770 South Wilson Avenue, Pasadena, CA 91125, USA \\ Received 2014 May 15; accepted 2014 July 11; published 2014 August 4
}

\begin{abstract}
Almost half of the stellar systems in the solar neighborhood are made up of multiple stars. In multiple-star systems, planet formation is under the dynamical influence of stellar companions, and the planet occurrence rate is expected to be different from that of single stars. There have been numerous studies on the planet occurrence rate of single star systems. However, to fully understand planet formation, the planet occurrence rate in multiple-star systems needs to be addressed. In this work, we infer the planet occurrence rate in multiple-star systems by measuring the stellar multiplicity rate for planet host stars. For a subsample of 56 Kepler planet host stars, we use adaptive optics (AO) imaging and the radial velocity (RV) technique to search for stellar companions. The combination of these two techniques results in high search completeness for stellar companions. We detect 59 visual stellar companions to 25 planet host stars with AO data. Three stellar companions are within $2^{\prime \prime}$ and 27 within $6^{\prime \prime}$. We also detect two possible stellar companions (KOI 5 and KOI 69) showing long-term RV acceleration. After correcting for a bias against planet detection in multiple-star systems due to flux contamination, we find that planet formation is suppressed in multiple-star systems with separations smaller than $1500 \mathrm{AU}$. Specifically, we find that compared to single star systems, planets in multiple-star systems occur $4.5 \pm 3.2,2.6 \pm 1.0$, and $1.7 \pm 0.5$ times less frequently when a stellar companion is present at a distance of 10,100 , and $1000 \mathrm{AU}$, respectively. This conclusion applies only to circumstellar planets; the planet occurrence rate for circumbinary planets requires further investigation.
\end{abstract}

Key words: methods: observational - methods: statistical - planetary systems - planets and satellites: fundamental parameters - techniques: high angular resolution - techniques: photometric

Online-only material: color figures

\section{INTRODUCTION}

The majority of the stars in the solar neighborhood belong to multiple-star systems (Duquennoy \& Mayor 1991; Fischer \& Marcy 1992; Raghavan et al. 2010; Duchêne \& Kraus 2013). In multiple-star systems, many planets have been detected. Some planets are detected in circumbinary orbits (P-type; Dvorak 1982), where the planet orbits both stars (e.g., Doyle et al. 2011; Welsh et al. 2012; Schwamb et al. 2013). Some others are detected in circumstellar orbits (S-type), where the planet orbits only one of the stars (e.g., Cochran et al. 1997; Eggenberger et al. 2004). Compared to our statistical knowledge of planets around single stars (Cumming et al. 2008; Howard et al. 2010; Mayor et al. 2011; Wright et al. 2012; Mann et al. 2012; Dressing \& Charbonneau 2013; Gaidos 2013; Swift et al. 2013; Kopparapu 2013; Petigura et al. 2013; Petigura et al. 2013; Bonfils et al. 2013; Parker \& Quanz 2013), our understanding of planet formation in multiple-star systems is rather limited; the planet occurrence rate in multiple-star systems is still largely unconstrained.

Planets in multiple-star systems can be studied by either searching for planets in known multiple-star systems, or searching for stellar companions in known planetary systems. There have been a few studies to search for planets in known multiplestar systems (e.g., Konacki 2005; Eggenberger \& Udry 2007; Konacki et al. 2009; Toyota et al. 2009). However, this direct method is prone to flux contamination of stellar companions, which affects measurement precision (Wright et al. 2012). In comparison, the technical challenges are dramatically reduced for detecting stellar companions in known planetary systems; it is easier to search for a star than it is to search for a planet.
Determination of the stellar multiplicity rate for planet host stars solves the inverse problem of measuring the planet occurrence rate in multiple-star systems (Wang et al. 2014). If planet host stars are rarely in multiple-star systems, this would indicate a low planet occurrence rate in these systems.

There have been numerous studies that have measured the stellar multiplicity rate of planet host stars. Most of these studies used imaging techniques, such as the adaptive optics (AO) imaging (Luhman \& Jayawardhana 2002; Patience et al. 2002; Eggenberger \& Udry 2007; Eggenberger et al. 2011; Adams et al. 2012, 2013; Law et al. 2013; Dressing et al. 2014), Lucky Imaging (Daemgen et al. 2009; Ginski et al. 2012; Lillo-Box et al. 2012; Bergfors et al. 2013; Lillo-Box et al. 2014), speckle imaging (Horch et al. 2012; Kane et al. 2014), wide field imaging (Mugrauer et al. 2007; Mugrauer \& Neuhäuser 2009), Hubble Space Telescope imaging (Gilliland et al. 2014), and other techniques (Raghavan et al. 2006, 2010; Roell et al. 2012). These studies have mostly reached similar conclusions that the stellar multiplicity rate of planet host stars is lower than or comparable to that for field stars in the solar neighborhood. Among these studies, some focused on stars hosting planets detected in ground-based radial velocity (RV) or transiting surveys (Luhman \& Jayawardhana 2002; Patience et al. 2002; Eggenberger et al. 2004; Raghavan et al. 2006; Mugrauer et al. 2007; Eggenberger \& Udry 2007; Daemgen et al. 2009; Mugrauer \& Neuhäuser 2009; Raghavan et al. 2010; Roell et al. 2012; Ginski et al. 2012; Bergfors et al. 2013; Knutson et al. 2014). However, the bias of ground-based planet surveys is difficult to assess due to an unknown threshold for excluding multiple-star systems. 
In comparison, the Kepler mission (Borucki et al. 2011; Batalha et al. 2013; Burke et al. 2014) did not strongly bias against multiple-star systems: (1) the low-angular-resolution Kepler Input Catalog images (Brown et al. 2011) did not reveal close binaries; (2) multiple-star systems (e.g., eclipsing binaries) received continued observation after detection. Therefore, the bias of ground-based surveys is not a major concern for studies of Kepler planet host stars (Lillo-Box et al. 2012; Adams et al. 2012; Horch et al. 2012; Adams et al. 2013; Law et al. 2013; Dressing et al. 2014; Kane et al. 2014; Gilliland et al. 2014; Lillo-Box et al. 2014). However, there is a detection bias against transiting planets in multiple-star systems. The transit depth is shallower due to the additional flux from stellar companions, which makes planet detection more difficult. This bias has to be taken into consideration when measuring the stellar multiplicity rate for planet host stars.

It is commonly accepted that planet formation may be disrupted in multiple-star systems with small separations (e.g., $\sim 10-200 \mathrm{AU})$. This is supported by both simulations (Thébault et al. 2006; Jang-Condell 2007; Quintana et al. 2007; Paardekooper et al. 2008; Kley \& Nelson 2008; Xie et al. 2010; Thébault 2011) and observations (Desidera \& Barbieri 2007; Bonavita \& Desidera 2007; Kraus et al. 2012). Therefore, surveys for gravitationally bound stellar companions around planet host stars provide the best path for understanding planet formation in multiple-star systems. High-resolution imaging techniques are efficient for separations greater than 0 '. 1 , and spectroscopic measurements are efficient at detecting stellar companions at smaller separations.

To carry out this work, we select a sample of 56 stars hosting planet candidates from the Kepler mission to search for potential stellar companions using the RV and the $\mathrm{AO}$ imaging techniques. The RV technique is sensitive to shortperiod stellar companions, and the AO technique is sensitive to those further out. The combination of these two techniques is sensitive to a larger range of semi-major axes, and results in a survey with much higher completeness than previous studies. We consider the detection bias against transiting planets in multiple-star systems, and correct for this when calculating the stellar multiplicity rate for planet host stars. We emphasize that we only consider planets in S-type circumstellar orbits.

The paper is organized as follows. We describe our sample and the sources for their RV and AO data in Section 2. In Section 3, we present the analyses of available data: searching for stellar companions to planet host stars using the RV and AO techniques. In Section 4, we introduce a method of correcting for detection bias against planets in multiple-star systems, and apply it to the measurement of stellar multiplicity rate for planet host stars. We then calculate the planet occurrence rate for single and multiple star systems by comparing their multiplicity rates. Discussion and summary are given in Section 5.

\section{SAMPLE DESCRIPTION AND DATA SOURCES}

$\mathrm{RV}$ and $\mathrm{AO}$ data are provided by the Kepler Community Follow-up Observation Program ${ }^{4}$ (CFOP). Since RV data are critical for probing stellar companions on close orbits, we select 56 Kepler Objects of Interest (KOIs) with at least three RV data points, for which the long-term RV acceleration due to a stellar companion may be measured. The RV data were taken with the HIRES instrument (Vogt et al. 1994) at the Keck I telescope, and reported in Marcy et al. (2014).

\footnotetext{
4 https://cfop.ipac.caltech.edu
}

The AO data for these $56 \mathrm{KOIs}$ were taken at different observatories including Keck, MMT, Gemini, Lick, Palomar, and WIYN. A summary of the sample and data sources is given in Table 1. Information on KOIs is provided by the NASA Exoplanet Archive (NEA; Huber et al. 2014). ${ }^{5}$ All the stars in our sample are solar-type stars with effective temperature $\left(T_{\text {eff }}\right)$ in the range between $4725 \mathrm{~K}$ and $6300 \mathrm{~K}$, and surface gravity $(\log g)$ in the range of 3.9 and 4.7. There are 27 (48\% of the sample) multi-planet systems.

\section{DETECTIONS AND CONSTRAINTS ON STELLAR COMPANIONS TO PLANET HOST STARS}

We use three techniques to detect and constrain stellar companions around planet host stars: the RV technique (Section 3.1), the AO imaging technique (Section 3.2), and the dynamical analysis (DA, Section 3.3). These three techniques are complementary and sensitive to different parts of parameter space. The $\mathrm{RV}$ technique is sensitive to close-in companions with small to intermediate mutual inclinations with respect to the planet orbital planes; the DA technique is sensitive to companions at large mutual inclinations; and the AO imaging technique is sensitive to stellar companions at larger separations. We will discuss in this section how we use these techniques to detect stellar companions and constrain their presence.

\subsection{RV Detections and Completeness}

We use the Keplerian Fitting Made Easy package (Giguere et al. 2012) to analyze the RV data. The procedure are described as follows. We first calculate the root mean square $\left(\mathrm{RMS}_{1}\right.$ in Table 2) of the RV data. If $\mathrm{RMS}_{1}$ is five times higher than the median reported RV uncertainties, $\delta v$, then we mark a variability flag. For systems marked with variability flags, we first fit the RV data with a linear trend, or a long-period orbit due to a nontransiting object. The systems with a significant linear trend $(3 \sigma)$ or the signal of an additional nontransiting object will be marked with a slope flag or a nontransiting component flag. The linear trend or the long-period orbit is then removed.

For the RV residuals after removing the linear trend or the long-period orbit, and the RVs for systems with no variability flag, we consider two cases. First, if the system has only one detected KOI, then we fit the RV data with a circular Keplerian orbit allowing only the RV semi-amplitude to change. If the resulting RMS $\left(\mathrm{RMS}_{2}\right)$ is smaller than $R M S_{1}$, then $R M S_{2}$ is used in subsequent analyses; otherwise $\mathrm{RMS}_{1}$ is used. Second, if the system has multiple KOIs, then we choose the one KOI causing the largest RV variation. When deciding which planet in the KOI causes the largest RV variation, we assume the planet mass-radius relationship from Lissauer et al. (2011), and calculate the nominal RV amplitude for each planet. Then, we fit the RV data with a circular Keplerian orbit allowing only the RV semi-amplitude to change. The minimum of $\mathrm{RMS}_{1}$ and $\mathrm{RMS}_{2}$ is used in subsequent analyses. In the above process, fitting eccentric orbit does not significantly change the RMS. In addition, including more KOIs for multi-planet systems does not always help to reduce the RMS because of large RV measurement uncertainty relative to the small RV signals of small planets.

Among 56 stars, only one shows a long-term RV slope: KOI 69 has an RV linear trend of $12.2 \pm 0.2 \mathrm{~ms}^{-1} \mathrm{yr}^{-1}$. During $4.1 \mathrm{yr}$ of RV measurements, there is no sign of deviation from

\footnotetext{
5 http://exoplanetarchive.ipac.caltech.edu/
} 
Table 1

$\mathrm{RV}$ and AO Data for $56 \mathrm{KOIs}$

\begin{tabular}{|c|c|c|c|c|c|c|c|c|c|c|c|c|}
\hline \multicolumn{8}{|c|}{ KOI } & \multicolumn{3}{|c|}{$\mathrm{RV}$} & \multicolumn{2}{|l|}{$\mathrm{AO}$} \\
\hline KOI & $\mathrm{KIC}$ & $\begin{array}{c}\alpha \\
(\mathrm{deg})\end{array}$ & $\begin{array}{c}\delta \\
(\mathrm{deg})\end{array}$ & $\begin{array}{c}K_{\mathrm{P}} \\
(\mathrm{mag})\end{array}$ & $\begin{array}{l}T_{\text {eff }} \\
(\mathrm{K})\end{array}$ & $\begin{array}{l}\log g \\
(\mathrm{cgs})\end{array}$ & $\# P L$ & $\begin{array}{c}T_{\text {start }} \\
\text { (MJD) }\end{array}$ & $\begin{array}{c}T_{\text {end }} \\
\text { (MJD) }\end{array}$ & \#RV & Telescope & Band \\
\hline 00005 & 8554498 & 289.739716 & 44.647419 & 11.665 & 5861.00 & 4.190 & 2 & 54983.516 & 56486.440 & 21 & Keck Palomar & $\mathrm{Br}-\gamma \mathrm{J}$ \\
\hline 00007 & 11853905 & 285.615326 & 50.135750 & 12.211 & 5858.00 & 4.280 & 1 & 55041.491 & 56134.480 & 22 & Keck Palomar & $\mathrm{H} \mathrm{J}$ \\
\hline 00010 & 6922244 & 281.288116 & 42.451080 & 13.563 & 6025.00 & 4.110 & 1 & 54983.540 & 55781.534 & 50 & Palomar & $\mathrm{J}$ \\
\hline 00017 & 10874614 & 296.837250 & 48.239944 & 13.303 & 5826.00 & 4.420 & 1 & 54984.561 & 55043.520 & 10 & Palomar & $\mathrm{J}$ \\
\hline 00018 & 8191672 & 299.407013 & 44.035053 & 13.369 & 6297.00 & 3.990 & 1 & 54985.594 & 55110.314 & 9 & Gemini Gemini Palomar & R Y J \\
\hline 00020 & 11804465 & 286.243439 & 50.040379 & 13.438 & 6011.00 & 4.230 & 1 & 55014.412 & 55761.325 & 16 & Palomar & $\mathrm{J}$ \\
\hline 00022 & 9631995 & 282.629669 & 46.323360 & 13.435 & 5972.00 & 4.410 & 1 & 55014.403 & 55792.438 & 16 & Palomar & $\mathrm{J}$ \\
\hline 00041 & 6521045 & 291.385986 & 41.990269 & 11.197 & 5909.00 & 4.300 & 3 & 54988.511 & 56533.359 & 64 & Keck Palomar & $\mathrm{Br}-\gamma \mathrm{J}$ \\
\hline 00069 & 3544595 & 291.418304 & 38.672359 & 9.931 & 5593.00 & 4.510 & 1 & 55042.587 & 56547.339 & 61 & Keck MMT MMT Palomar & $\mathrm{Br}-\gamma \mathrm{J}$ K J \\
\hline 00070 & 6850504 & 287.697998 & 42.338718 & 12.498 & 5443.00 & 4.450 & 5 & 55073.386 & 56533.484 & 38 & Palomar & $\mathrm{J}$ \\
\hline 00072 & 11904151 & 285.679382 & 50.241299 & 10.961 & 5627.00 & 4.390 & 2 & 55073.400 & 56172.301 & 54 & MMT MMT Palomar & $\mathrm{J}$ K J \\
\hline 00082 & 10187017 & 281.482727 & 47.208031 & 11.492 & 4908.00 & 4.610 & 5 & 55311.579 & 56533.335 & 65 & Keck MMT MMT & $\mathrm{Br}-\gamma \mathrm{J} \mathrm{K}$ \\
\hline 00084 & 2571238 & 290.420807 & 37.851799 & 11.898 & 5541.00 & 4.530 & 1 & 55073.419 & 55723.450 & 20 & Palomar & $\mathrm{J}$ \\
\hline 00085 & 5866724 & 288.688690 & 41.151180 & 11.018 & 6172.00 & 4.360 & 3 & 55696.490 & 55738.516 & 6 & MMT MMT & $\mathrm{J}$ K \\
\hline 00087 & 10593626 & 289.217499 & 47.884460 & 11.664 & 5510.00 & 4.500 & 1 & 55425.386 & 56521.486 & 26 & Palomar Palomar & J K \\
\hline 00103 & 2444412 & 291.683350 & 37.751591 & 12.593 & 5531.00 & 4.440 & 1 & 55073.441 & 55797.539 & 16 & Palomar & $\mathrm{J}$ \\
\hline 00104 & 10318874 & 281.194733 & 47.497131 & 12.895 & 4786.00 & 4.590 & 1 & 55377.342 & 56525.316 & 30 & Keck Palomar Palomar & K J K \\
\hline 00108 & 4914423 & 288.984558 & 40.064529 & 12.287 & 5975.00 & 4.330 & 2 & 55073.469 & 56145.498 & 22 & Keck Palomar & K J \\
\hline 00111 & 6678383 & 287.604614 & 42.166779 & 12.596 & 5711.00 & 4.410 & 4 & 55372.555 & 56521.442 & 14 & Palomar & $\mathrm{J}$ \\
\hline 00116 & 8395660 & 300.863983 & 44.337551 & 12.882 & 5865.00 & 4.410 & 4 & 55133.397 & 56532.332 & 33 & Keck MMT & K K \\
\hline 00122 & 8349582 & 284.482452 & 44.398041 & 12.346 & 5714.00 & 4.390 & 1 & 55073.495 & 56151.511 & 33 & Keck Palomar & K J \\
\hline 00123 & 5094751 & 290.392731 & 40.284870 & 12.365 & 5871.00 & 4.150 & 2 & 55074.492 & 56166.345 & 15 & Keck Palomar & K J \\
\hline 00137 & 8644288 & 298.079437 & 44.746319 & 13.549 & 5385.00 & 4.430 & 3 & 55075.509 & 56146.484 & 20 & Palomar & $\mathrm{J}$ \\
\hline 00148 & 5735762 & 299.139221 & 40.949020 & 13.040 & 5190.00 & 4.490 & 3 & 55073.527 & 56532.436 & 43 & Keck Palomar & K J \\
\hline 00153 & 12252424 & 287.997894 & 50.944328 & 13.461 & 4725.00 & 4.640 & 2 & 55313.592 & 56524.353 & 29 & Keck MMT Palomar Palomar & $\mathrm{Br}-\gamma \mathrm{K} \mathrm{J} \mathrm{K}$ \\
\hline 00157 & 6541920 & 297.115112 & 41.909142 & 13.709 & 5685.00 & 4.380 & 6 & 55440.501 & 56533.434 & 7 & Palomar Robo-AO & LP600 \\
\hline 00180 & 9573539 & 284.394287 & 46.249081 & 13.024 & 5680.00 & 4.500 & 1 & 55074.466 & 55083.341 & 6 & Palomar & $\mathrm{J}$ \\
\hline 00244 & 4349452 & 286.638397 & 39.487881 & 10.734 & 6103.00 & 4.070 & 2 & 55366.603 & 56519.408 & 104 & Keck Palomar Palomar & $\mathrm{Br}-\gamma \mathrm{J} \mathrm{K}$ \\
\hline 00245 & 8478994 & 284.059540 & 44.518215 & 9.705 & 5288.00 & 4.590 & 4 & 55312.586 & 56523.237 & 59 & Gemini Gemini Keck MMT MMT Palomar & R Y Br- $\gamma \mathrm{J} \mathrm{K} \mathrm{K}$ \\
\hline 00246 & 11295426 & 291.032318 & 49.040272 & 9.997 & 5793.00 & 4.281 & 2 & 55312.582 & 56519.420 & 65 & Keck MMT MMT & $\mathrm{Br}-\gamma \mathrm{J} \mathrm{K}$ \\
\hline 00261 & 5383248 & 297.069611 & 40.525131 & 10.297 & 5692.00 & 4.420 & 1 & 55404.530 & 56518.546 & 36 & Keck MMT MMT & $\mathrm{Br}-\gamma \mathrm{J} \mathrm{K}$ \\
\hline 00263 & 10514430 & 281.273804 & 47.774399 & 10.821 & 5820.00 & 4.150 & 1 & 55395.529 & 55788.482 & 6 & MMT MMT Palomar Palomar & J K J K \\
\hline 00265 & 12024120 & 297.018829 & 50.408981 & 11.994 & 6040.00 & 4.360 & 1 & 55782.522 & 56532.354 & 27 & Gemini Gemini Palomar Palomar & R Y J K \\
\hline
\end{tabular}


Table 1

(Continued)

\begin{tabular}{|c|c|c|c|c|c|c|c|c|c|c|c|c|}
\hline \multicolumn{8}{|c|}{ KOI } & \multicolumn{3}{|c|}{$\mathrm{RV}$} & \multicolumn{2}{|l|}{$\mathrm{AO}$} \\
\hline KOI & KIC & $\begin{array}{c}\alpha \\
(\mathrm{deg})\end{array}$ & $\begin{array}{c}\delta \\
(\mathrm{deg})\end{array}$ & $\begin{array}{c}K_{\mathrm{P}} \\
(\mathrm{mag})\end{array}$ & $\begin{array}{l}T_{\text {eff }} \\
(\mathrm{K})\end{array}$ & $\begin{array}{l}\log g \\
(\operatorname{cgs})\end{array}$ & \#PL & $\begin{array}{c}T_{\text {start }} \\
\text { (MJD) }\end{array}$ & $\begin{array}{c}T_{\text {end }} \\
\text { (MJD) }\end{array}$ & $\# \mathrm{RV}$ & Telescope & Band \\
\hline 00273 & 3102384 & 287.478516 & 38.228840 & 11.457 & 5783.00 & 4.430 & 1 & 55431.309 & 56613.223 & 20 & MMT MMT & J K \\
\hline 00274 & 8077137 & 282.492218 & 43.980209 & 11.390 & 6090.00 & 4.130 & 2 & 55403.447 & 56474.522 & 8 & Gemini Gemini MMT MMT & R Y J K \\
\hline 00283 & 5695396 & 288.530823 & 40.942299 & 11.525 & 5687.00 & 4.420 & 2 & 55433.368 & 56524.331 & 30 & Keck Palomar Palomar & $\mathrm{Br}-\gamma \mathrm{J} \mathrm{K}$ \\
\hline 00289 & 10386922 & 282.945648 & 47.574905 & 12.747 & 5812.00 & 4.458 & 2 & 56449.401 & 56532.291 & 5 & Lick & $\mathrm{J}$ \\
\hline 00292 & 11075737 & 287.326630 & 48.673431 & 12.872 & 5780.00 & 4.430 & 1 & 55377.475 & 56166.411 & 21 & Keck Palomar Palomar & K J K \\
\hline 00299 & 2692377 & 285.661652 & 37.964500 & 12.899 & 5538.00 & 4.340 & 1 & 55403.512 & 56530.414 & 32 & Keck & $\mathrm{K}$ \\
\hline 00305 & 6063220 & 297.354004 & 41.300049 & 12.970 & 4782.00 & 4.610 & 1 & 55403.543 & 56531.340 & 36 & Keck & K \\
\hline 00321 & 8753657 & 291.848053 & 44.968220 & 12.520 & 5538.00 & 4.340 & 2 & 55378.534 & 56493.368 & 47 & Keck Lick & K J \\
\hline 00364 & 7296438 & 295.872314 & 42.881149 & 10.087 & 5798.00 & 4.240 & 1 & 55376.346 & 55699.442 & 3 & WIYN & $i$ \\
\hline 00365 & 11623629 & 297.486908 & 49.623451 & 11.195 & 5451.00 & 4.490 & 2 & 55402.354 & 56532.377 & 24 & Palomar Palomar & J K \\
\hline 00377 & 3323887 & 285.573975 & 38.400902 & 13.803 & 5777.00 & 4.450 & 3 & 55342.448 & 56506.363 & 16 & Palomar Palomar & $\mathrm{J} \mathrm{K}$ \\
\hline 00701 & 9002278 & 283.212738 & 45.349861 & 13.725 & 4807.00 & 4.690 & 5 & 56137.475 & 56507.530 & 15 & Keck & $\mathrm{K}$ \\
\hline 00975 & 3632418 & 287.361816 & 38.714016 & 8.224 & 6131.00 & 3.950 & 1 & 55439.438 & 56486.562 & 44 & MMT MMT & $\mathrm{J}$ K \\
\hline 01431 & 11075279 & 287.022278 & 48.681938 & 13.460 & 5649.00 & 4.460 & 1 & 56472.391 & 56532.502 & 6 & WIYN & $i$ \\
\hline 01439 & 11027624 & 290.851776 & 48.521339 & 12.849 & 5930.00 & 4.090 & 1 & 55075.273 & 56531.312 & 6 & Lick & $\mathrm{J}$ \\
\hline 01442 & 11600889 & 286.036346 & 49.614510 & 12.521 & 5476.00 & 4.448 & 1 & 55696.518 & 56446.424 & 17 & Keck Lick & K J \\
\hline 01463 & 7672940 & 288.258636 & 43.376465 & 12.328 & 6020.00 & 4.380 & 1 & 56027.511 & 56530.292 & 3 & WIYN & $i$ \\
\hline 01612 & 10963065 & 284.786194 & 48.423229 & 8.769 & 6027.00 & 4.220 & 1 & 55697.588 & 56494.420 & 42 & Keck Lick & $\mathrm{Br}-\gamma \mathrm{J}$ \\
\hline 01781 & 11551692 & 287.605591 & 49.523258 & 12.231 & 4977.00 & 4.590 & 3 & 56076.566 & 56112.331 & 9 & Palomar Robo-AO & LP600 \\
\hline 01925 & 9955598 & 293.679199 & 46.852760 & 9.439 & 5365.00 & 4.430 & 1 & 55999.646 & 56547.332 & 40 & Keck Palomar & $\mathrm{Br}-\gamma \mathrm{K}$ \\
\hline 02169 & 9006186 & 285.207489 & 45.384350 & 12.404 & 5447.00 & 4.420 & 4 & 56099.455 & 56171.456 & 4 & Palomar Robo-AO & LP600 \\
\hline 02687 & 7202957 & 292.615112 & 42.764252 & 10.158 & 5803.00 & 3.910 & 2 & 55999.652 & 56531.383 & 22 & Palomar & $\mathrm{K}$ \\
\hline 02720 & 8176564 & 295.439667 & 44.039162 & 10.338 & 6109.00 & 4.410 & 1 & 56018.575 & 56519.389 & 18 & Keck Palomar & $\mathrm{Br}-\gamma \mathrm{K}$ \\
\hline
\end{tabular}


Table 2

RV Measurement Results for 56 KOIs

\begin{tabular}{|c|c|c|c|c|c|c|c|c|}
\hline KOI & KIC & $\begin{array}{l}\mathrm{RMS}_{1}{ }^{\mathrm{b}} \\
\left(\mathrm{m} \mathrm{s}^{-1}\right)\end{array}$ & $\begin{array}{c}\delta v \\
\left(\mathrm{~m} \mathrm{~s}^{-1}\right)\end{array}$ & $\frac{\mathrm{RMS}_{1}}{\delta v}>5$ & Slope & Non-transiting & $\begin{array}{l}\mathrm{RMS}_{2}{ }^{\mathrm{c}} \\
\left(\mathrm{m} \mathrm{s}^{-1}\right)\end{array}$ & $\frac{\mathrm{RMS}_{2}}{\delta v}>5$ \\
\hline $00005^{\mathrm{a}}$ & 8554498 & 94.9 & 4.2 & $\sqrt{ }$ & & $\sqrt{ }$ & 23.7 & $\sqrt{ }$ \\
\hline 00007 & 11853905 & 7.9 & 2.1 & & & & 4.5 & \\
\hline 00010 & 6922244 & 53.8 & 13.2 & & & & 47.1 & \\
\hline 00017 & 10874614 & 57.8 & 3.6 & $\sqrt{ }$ & & & 7.1 & \\
\hline 00018 & 8191672 & 178.1 & 6.1 & $\sqrt{ }$ & & & 18.2 & \\
\hline 00020 & 11804465 & 44.5 & 4.1 & $\sqrt{ }$ & & & 22.0 & $\sqrt{ }$ \\
\hline 00022 & 9631995 & 44.2 & 3.8 & $\sqrt{ }$ & & & 40.9 & $\sqrt{ }$ \\
\hline 00041 & 6521045 & 5.4 & 1.6 & & & & 5.4 & \\
\hline $00069^{a}$ & 3544595 & 21.2 & 1.3 & $\sqrt{ }$ & $\sqrt{ }$ & & 3.0 & \\
\hline 00070 & 6850504 & 11.0 & 1.9 & $\sqrt{ }$ & & & 10.2 & $\sqrt{ }$ \\
\hline 00072 & 11904151 & 4.6 & 1.6 & & & & 3.7 & \\
\hline 00082 & 10187017 & 4.5 & 1.2 & & & & 4.2 & \\
\hline 00084 & 2571238 & 11.2 & 1.8 & $\sqrt{ }$ & & & 11.2 & $\sqrt{ }$ \\
\hline 00085 & 5866724 & 10.1 & 1.9 & $\sqrt{ }$ & & & 15.5 & $\sqrt{ }$ \\
\hline 00087 & 10593626 & 5.0 & 1.6 & & & & 4.8 & \\
\hline 00103 & 2444412 & 6.8 & 1.9 & & & & 6.2 & \\
\hline 00104 & 10318874 & 96.1 & 2.0 & $\sqrt{ }$ & & $\sqrt{ }$ & 6.1 & \\
\hline 00108 & 4914423 & 7.6 & 2.2 & & & & 7.4 & \\
\hline 00111 & 6678383 & 6.1 & 2.6 & & & & 6.6 & \\
\hline 00116 & 8395660 & 6.5 & 2.4 & & & & 6.5 & \\
\hline 00122 & 8349582 & 5.1 & 1.7 & & & & 4.7 & \\
\hline 00123 & 5094751 & 7.1 & 2.4 & & & & 6.9 & \\
\hline 00137 & 8644288 & 9.7 & 2.5 & & & & 8.0 & \\
\hline 00148 & 5735762 & 32.7 & 2.3 & $\sqrt{ }$ & & $\sqrt{ }$ & 14.2 & $\sqrt{ }$ \\
\hline 00153 & 12252424 & 9.7 & 2.4 & & & & 8.9 & \\
\hline 00157 & 6541920 & 36.7 & 8.1 & & & & 49.9 & $\sqrt{ }$ \\
\hline 00180 & 9573539 & 15.7 & 2.1 & $\sqrt{ }$ & & & 15.6 & $\sqrt{ }$ \\
\hline 00244 & 4349452 & 8.3 & 3.5 & & & $\sqrt{ }$ & 6.5 & \\
\hline 00245 & 8478994 & 5.0 & 1.3 & & & & 3.3 & \\
\hline 00246 & 11295426 & 16.9 & 1.3 & $\sqrt{ }$ & & $\sqrt{ }$ & 3.6 & \\
\hline 00261 & 5383248 & 5.2 & 1.5 & & & & 5.3 & \\
\hline 00263 & 10514430 & 11.1 & 3.2 & & & & 13.9 & \\
\hline 00265 & 12024120 & 4.7 & 1.8 & & & & 4.5 & \\
\hline 00273 & 3102384 & 91.7 & 1.4 & $\sqrt{ }$ & & & 10.3 & $\sqrt{ }$ \\
\hline 00274 & 8077137 & 4.0 & 1.9 & & & & 3.7 & \\
\hline 00283 & 5695396 & 5.7 & 1.6 & & & & 5.7 & \\
\hline 00289 & 10386922 & 12.1 & 2.1 & $\sqrt{ }$ & & & 12.1 & $\sqrt{ }$ \\
\hline 00292 & 11075737 & 6.2 & 2.3 & & & & 4.5 & \\
\hline 00299 & 2692377 & 6.6 & 1.9 & & & & 6.0 & \\
\hline 00305 & 6063220 & 5.8 & 1.7 & & & & 5.6 & \\
\hline 00321 & 8753657 & 4.3 & 1.7 & & & & 4.0 & \\
\hline 00364 & 7296438 & 51.3 & 0.3 & $\sqrt{ }$ & & & 51.3 & $\sqrt{ }$ \\
\hline 00365 & 11623629 & 4.3 & 1.3 & & & & 4.0 & \\
\hline 00377 & 3323887 & 13.3 & 6.0 & & & & 12.3 & \\
\hline 00701 & 9002278 & 4.7 & 3.0 & & & & 4.9 & \\
\hline 00975 & 3632418 & 8.9 & 3.0 & & & & 8.9 & \\
\hline 01431 & 11075279 & 8.3 & 2.1 & & & & 7.1 & \\
\hline 01439 & 11027624 & 10.2 & 2.3 & & & & 15.4 & $\sqrt{ }$ \\
\hline 01442 & 11600889 & 89.0 & 2.0 & $\sqrt{ }$ & & $\sqrt{ }$ & 3.1 & \\
\hline 01463 & 7672940 & 116.1 & 2.8 & $\sqrt{ }$ & & & 116.1 & $\sqrt{ }$ \\
\hline 01612 & 10963065 & 4.1 & 1.5 & & & & 4.0 & \\
\hline 01781 & 11551692 & 21.7 & 1.4 & $\sqrt{ }$ & & & 24.3 & $\sqrt{ }$ \\
\hline 01925 & 9955598 & 3.4 & 1.1 & & & & 2.5 & \\
\hline 02169 & 9006186 & 6.6 & 0.9 & $\sqrt{ }$ & & & 6.6 & $\sqrt{ }$ \\
\hline 02687 & 7202957 & 9.2 & 1.3 & $\sqrt{ }$ & & & 10.2 & $\sqrt{ }$ \\
\hline 02720 & 8176564 & 3.8 & 1.4 & & & & 3.6 & \\
\hline
\end{tabular}

Notes.

${ }^{a}$ KOIs considered in multiple-star systems. See Sections 3.2.4 and 3.2.5 for detailed discussions.

b RMS of the RV measurements.

${ }^{c}$ RMS after removing the linear trend or the long-period signal and the RV signal caused by detected planet candidates. 


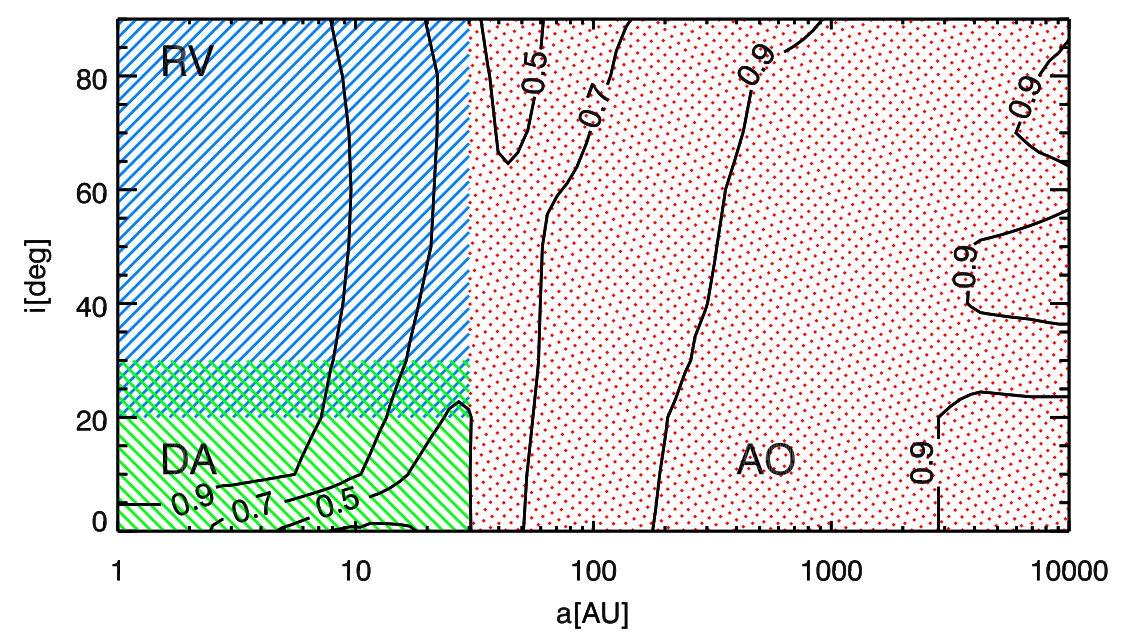

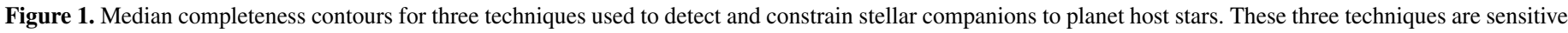

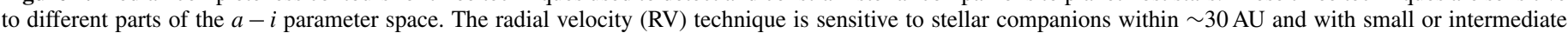

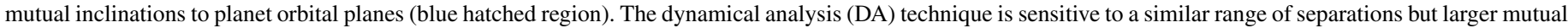

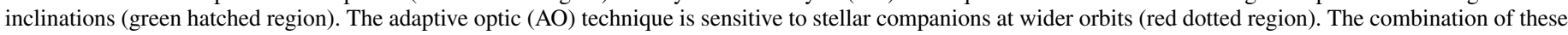
three techniques contributes to a survey of stellar companions with high completeness.

(A color version of this figure is available in the online journal.)

the linear trend, indicating that the companion is at least $4 \mathrm{AU}$ away. More discussion regarding this system will be given in Section 3.2 after considering the AO data. Six systems have nontransiting objects revealed by the RV data: KOI 5, KOI 104, KOI 148, KOI 244, KOI 246, and KOI 1442. The latter five are known nontransiting planets (Marcy et al. 2014). KOI 5 shows a parabolic acceleration, but the period of the nontransiting object is unconstrained. We will discuss KOI 5 more in Section 3.2 with the addition of AO data. For 15 cases, RMS is still five times higher than the reported RV measurement uncertainty after considering a nontransiting object, or the KOI planet dominating the RV variability. The "excessive" RV variability may be attributed to the following factors or their combinations: very limited number of RV data points, an underestimated RV measurement uncertainty, excessive stellar activity, and additional stellar or planetary components. We find that 12 out of the 15 KOIs with "excessive" RV variation have fewer than $21 \mathrm{RV}$ measurements, which is the median number of RV measurements for the $56 \mathrm{KOIs}$ in our sample. Seven of them have fewer than $10 \mathrm{RV}$ measurements. The limited number of $\mathrm{RV}$ measurements would result in an improper RV orbital fitting, which leads to a higher RMS. In addition, RV jitter is not considered in the reported RV uncertainty in Table 2. After considering a typical RV jitter of $1-3 \mathrm{~m} \mathrm{~s}^{-1}$ for Kepler stars with RV measurements (Marcy et al. 2014), 10 out of the 15 KOIs with "excessive" RV variation have less than five times of the RV uncertainty. KOI 22 remains the only KOI in our sample with "excessive" RV variation that cannot be explained by either the limited number of RV measurements or stellar activity.

We study the completeness of searching for stellar companions by simulations following the subsequent procedures. We first define a parameter space, $a-i$ space, where $a$ is the separation of a companion star, and $i$ is the mutual inclination of the sky plane and the companion star orbital plane. We divide the parameter space into many fine grids $\left(\Delta a=0.5 \mathrm{AU}, \Delta i=10^{\circ}\right)$. For each star, we simulate 1000 companion stars on each grid, and count how many simulated companion stars are detected given the time baseline, observation epochs, and measurement uncertainties of the RV data. Specifically, we generate a synthetic RV data set for each of the simulated companion stars.
Observation epochs and measurement uncertainties remain the same as the original RV data. If the RMS of the synthetic RV data is three times larger than the observed RV RMS, i.e., the smaller of $\mathrm{RMS}_{1}$ and $\mathrm{RMS}_{2}$, then we count the simulated stellar companion as a detection. The separation and mass ratio distributions of simulated stellar companions follow the normal distributions reported in Duquennoy \& Mayor (1991), i.e., $\log _{10} a=1.49, \sigma_{\log _{10} a}=1.54 ; q=m 2 / m 1=0.23, \sigma_{q}=$ 0.42 . We use the median orbital eccentricity for binary stars ( $e=0.4$; Duquennoy \& Mayor 1991) and a random periastron distribution in simulations. The median completeness contours are shown in Figure 1. RV completeness drops to below 50\% as separations become larger than $30 \mathrm{AU}$.

In summary, RV observations of 56 stars reveal seven nontransiting companions, five of these are previously reported planets (Marcy et al. 2014). Orbits of the other two are unconstrained because of limited RV baselines. KOI 5 shows a parabolic RV acceleration, and KOI 69 shows a linear RV trend of $12.2 \pm 0.2 \mathrm{~ms}^{-1} \mathrm{yr}^{-1}$. The nature of these two companions will be discussed more in the following section.

\subsection{AO Detections and Completeness}

The RV variation of most of stellar companions at larger separations is difficult to measure because of the long periodicity. However, the AO imaging technique is more effective in constraining stellar companions at larger separations. We will discuss in the following part how we detect and characterize stellar companions based on AO images.

\subsubsection{Contrast Curve}

The contrast curve of an image provides detection thresholds for detecting faint companions around a star. The procedures of calculating the contrast curve are described as follows. We define a series of concentric annuli, centered on the star, for which we calculate the median and the standard deviation of flux for pixels within these annuli. We use the value of five times the standard deviation above the median as the $5 \sigma$ detection limit. The contrast curve is the $5 \sigma$ detection limit as a function of the radii of concentric annuli. The median contrast curve and the $1 \sigma$ 


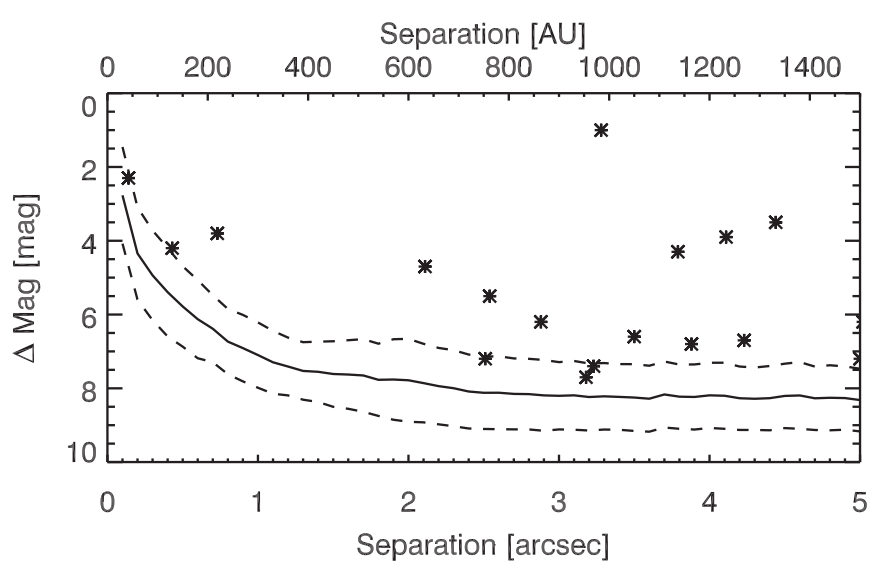

Figure 2. Median contrast curve for the AO images. Dashed lines are $1 \sigma$ deviations of the contrast curve. Detections within $5^{\prime \prime}$ are shown as asterisks. Physical projected separation in AU is calculated assuming the average distance of the sample, i.e., 300 pc. When analyzing the detection completeness, each star in our sample is treated individually for the observation band in which the AO image was taken. A total of 59 visual companions around 25 planet host stars are detected (Table 3).

deviation of the AO images we use in this paper are shown in Figure 2, where each pixel is converted into angular separation based on plate scale of each instrument: 0.010 pixel $^{-1}$ for Keck NIRC2 (Wizinowich et al. 2000), 0'.011 pixel $^{-1}$ for Gemini DSSI, ${ }^{6}{ }^{\prime \prime} .019$ pixel $^{-1}$ or 0 '.038 pixel $^{-1}$ for MMT ARIES (Sarlot et al. 1999), 0'.025 pixel $^{-1}$ for Palomar PHARO (Hayward et al. 2001), 0.'075 pixel $^{-1}$ for Lick IRCAL (Lloyd et al. 2000), $00^{\prime}$.017-0.'018 pixel $^{-1}$ for WIYN DSSI (Horch et al. 2009), and 0.'043 pixel $^{-1}$ for Palomar Robo-AO (Law et al. 2013).

\subsubsection{Distance Estimation}

In order to obtain the physical projected separation between detected companions and the central stars, we need to estimate the distance. The distance of a star can be measured with the distance modulus and an estimation of extinction. The extinction estimation in the $V$ band $\left(A_{V}\right)$ is obtained from the Mikulski Archive for Space Telescopes (MAST). ${ }^{7}$ Details of $A_{V}$ estimation can be found in Section 6 and Section 7 in Brown et al. (2011). The distance modulus is the magnitude difference between the apparent magnitude and the absolute magnitude in the $V$ band. The apparent $V$ magnitude is calculated based a conversion from $g^{\prime}$ and $r^{\prime}$ magnitudes (Smith et al. 2002). The absolute $V$ magnitude is estimated with the Yale-Yonsei (Y2) stellar evolution model (Demarque et al. 2004): with $T_{\text {eff }}$, $\log g$, age, and $[\mathrm{Fe} / \mathrm{H}]$ measured from spectroscopic and/or asteroseismic observations, the absolute $V$ magnitude can be estimated from the Y2 interpolator. For stars with an unknown $A_{V}$, which is the case for seven stars, we use the distance modulus in $K$ band to estimate the distance with the assumption that $K$-band extinction is much smaller than $V$ band for Kepler stars. Distances for KOIs with visual stellar companion detections are provided in Table 3.

\subsubsection{Detection and Completeness}

Based on the images from the CFOP, we detect a total of 59 visual stellar companions around 25 planet host stars (Table 3 ). Fourteen stars $(25 \%)$ have stellar companions within a $5^{\prime \prime}$ radius.

\footnotetext{
6 http://www.gemini.edu/sciops/instruments/dssi-speckle-camera-north

7 https://archive.stsci.edu/
}

The closest companion has a projected separation of $40.9 \mathrm{AU}$ (0.'14) from KOI 5.

The 56 stars in our sample have an average distance of $\sim 300$ pc. Given the contrast curve shown in Figure 2, the search for stellar companions closer than $\sim 40 \mathrm{AU}$ and low-mass stars $(\Delta \mathrm{Mag}>8)$ is not complete. We therefore conduct simulations to evaluate the completeness of the AO survey. Similar to the RV completeness simulations in Section 3.1, we artificially generate 1000 companion stars at each predefined grid in the $a-i$ parameter space. If the contrast ratio ( $\Delta \mathrm{Mag}$ ) between a simulation star and the central star is smaller than the value given by AO $5 \sigma$ contrast curve, then we record it as a detection. Note that the contrast curves used in simulations are those calculated for each individual star in the observed band rather than the median contrast curve shown in Figure 2. The AO completeness contours (median of 56 stars) are plotted in Figure 1. From this plot, we show that the AO completeness is less than $50 \%$ for separations smaller than $\sim 40 \mathrm{AU}$. At smaller separations, the RV technique becomes a much more efficient way of detecting stellar companions.

\subsubsection{KOI 5}

KOI 5 has a parabolic RV acceleration indicating a distant companion, but the orbit of this companion is unconstrained given only approximately four years of observation and poor phase coverage. There are many possible orbital solutions given the current RV data. Figure 3 shows two examples. If the $\mathrm{RV}$ acceleration is caused by the stellar companion detected by the AO imaging, then it requires a highly eccentric orbit $(e=0.92)$ to reasonably fit the RV data. We estimate the mass of the AO detected stellar companion to be $\sim 0.5 M_{\odot}$ based on its differential magnitude in the $K$ band (Kraus \& Hillenbrand 2007). Alternatively, the observed RV acceleration can be explained by a stellar companion $\left(0.08 M_{\odot}\right)$ at $7 \mathrm{AU}$ separation on a circular orbit. Any solutions with separations smaller than $7 \mathrm{AU}$ should involve companions that fall into substellar mass regime. Therefore, we conclude that a stellar companion may exist around KOI 5, but with a separation larger than 7 AU (i.e., 0'.024 angular separation).

$$
\text { 3.2.5. KOI } 69
$$

KOI 69 shows a linear RV trend of $12.2 \pm 0.2 \mathrm{~ms}^{-1} \mathrm{yr}^{-1}$, which can be caused either by a more distant star or a closer sub-stellar object. Figure 4 shows possible parameter space for this companion. RV data exclude any companions below the straight solid line because they are not massive enough to cause the trend. Although AO data shows nondetection for KOI 69, the AO contrast curve can put constraint on any bright stellar objects which would have been detected. After considering the constraints from $\mathrm{AO}$ and $\mathrm{RV}$ observations, if the companion causing the RV linear trend is a star, it is mostly likely to lie between 15.5 and 33.0 AU (i.e., 0 '.18 and 0.'38 in angular separation), and its mass cannot exceed 102 Jupiter mass $(2 \sigma)$. If the companion mass is in the substellar regime, its mass and separation is confined to a parallelogram marked as "Substellar" in Figure 4. The four vertices of the parallelogram are (5.5 AU, 10.0 $\left.M_{J}\right),\left(9.8 \mathrm{AU}, 10.0 M_{J}\right),\left(27.6 \mathrm{AU}, 80.0 M_{J}\right)$, and (15.5 AU, $\left.80.0 M_{J}\right)$.

\subsubsection{Visual Companions Association}

We detect 59 visual companions around 25 planet host stars, but we do not know whether they are associated or bonded to the 
Table 3

Visual Companion Detections with AO Data

\begin{tabular}{|c|c|c|c|c|c|c|}
\hline \multirow[t]{2}{*}{ KOI } & \multirow{2}{*}{$\begin{array}{l}\Delta \mathrm{Mag} \\
(\mathrm{mag})\end{array}$} & \multicolumn{2}{|c|}{ Separation } & \multirow{2}{*}{$\begin{array}{c}\text { Distance } \\
\text { (pc) }\end{array}$} & \multirow{2}{*}{$\begin{array}{c}\text { Significance } \\
(\sigma)\end{array}$} & \multirow{2}{*}{$\begin{array}{l}\text { P.A. } \\
\text { (deg) }\end{array}$} \\
\hline & & $(\operatorname{arcsec})$ & $(\mathrm{AU})$ & & & \\
\hline K00005 & $2.3(\mathrm{Br}-\gamma)$ & 0.14 & 40.9 & $290.9_{-19.4}^{+63.2}$ & 28.2 & 308.9 \\
\hline K00010 & $6.8(J)$ & 3.88 & 3663.9 & $944.5_{-139.1}^{+100.1}$ & 22.0 & 89.3 \\
\hline K00017 & $3.9(J)$ & 4.11 & 2130.7 & $517.9_{-28.4}^{+27.6}$ & 206.2 & 39.5 \\
\hline K00018 & $3.9(J)$ & 7.26 & 8241.0 & $1135.9_{-154.0}^{+84.4}$ & 323.2 & 148.2 \\
\hline K00018 & $6.3(J)$ & 9.68 & 10995.5 & $1135.9_{-154.0}^{+84.4}$ & 32.1 & 344.7 \\
\hline K00018 & $6.6(J)$ & 3.50 & 3971.8 & $1135.9_{-154.0}^{+84.4}$ & 28.5 & 110.1 \\
\hline K00018 & $7.3(J)$ & 5.09 & 5783.0 & $1135.9_{-154.0}^{+84.4}$ & 14.1 & 211.3 \\
\hline K00018 & $7.7(J)$ & 5.89 & 6693.3 & $1135.9_{-154.0}^{+84.4}$ & 9.8 & 106.3 \\
\hline K00018 & $7.7(J)$ & 10.82 & 12293.3 & $1135.9_{-154.0}^{+84.4}$ & 7.7 & 222.1 \\
\hline K00018 & $7.3(J)$ & 7.26 & 8241.0 & $1135.9_{-154.0}^{+84.4}$ & 7.3 & 77.6 \\
\hline K00018 & $8.0(J)$ & 9.69 & 11004.0 & $1135.9_{-154.0}^{+84.4}$ & 6.6 & 339.2 \\
\hline K00018 & $8.2(J)$ & 7.09 & 8059.2 & $1135.9_{-154.0}^{+84.4}$ & 6.5 & 219.2 \\
\hline K00070 & $4.3(J)$ & 3.79 & 1058.9 & $279.5_{-23.6}^{+25.3}$ & 217.7 & 51.8 \\
\hline K00087 & $6.2(J), 6.1(K)$ & 5.49 & 956.9 & $174.4_{-12.1}^{+15.2}$ & 78.2 & 177.2 \\
\hline K00087 & $7.4(J), 6.6(K)$ & 5.53 & 964.1 & $174.4_{-12.1}^{+15.2}$ & 28.4 & 75.2 \\
\hline K00103 & $7.3(J)$ & 9.81 & 2985.6 & $304.4_{-27.0}^{+29.0}$ & 9.5 & 278.5 \\
\hline K00108 & $5.5(J)$ & 9.52 & 3357.4 & $352.7_{-22.0}^{+36.0}$ & 98.8 & 348.6 \\
\hline K00108 & $7.2(J)$ & 5.00 & 1764.4 & $352.7_{-22.0}^{+36.0}$ & 21.5 & 112.5 \\
\hline K00108 & $7.2(J)$ & 2.51 & 887.0 & $352.7_{-22.0}^{+36.0}$ & 19.9 & 74.8 \\
\hline K00108 & $7.4(J)$ & 3.23 & 1139.2 & $352.7_{-22.0}^{+36.0}$ & 18.2 & 100.9 \\
\hline K00108 & $7.4(J)$ & 8.90 & 3139.4 & $352.7_{-22.0}^{+36.0}$ & 17.1 & 19.2 \\
\hline K00111 & $7.5(J)$ & 7.13 & 2052.6 & $297.8_{-29.1}^{+26.0}$ & 10.8 & 117.7 \\
\hline K00111 & $7.8(J)$ & 9.07 & 2702.5 & $297.8_{-29.1}^{+26.0}$ & 7.7 & 175.5 \\
\hline K00111 & $8.2(J)$ & 6.70 & 1995.1 & $297.8_{-29.1}^{+26.0}$ & 8.2 & 96.0 \\
\hline K00116 & $3.8(K)$ & 8.00 & 2907.0 & $363.2_{-40.3}^{+56.7}$ & 164.1 & 353.5 \\
\hline K00116 & $4.8(K)$ & 12.96 & 4707.6 & $363.2_{-40.3}^{+56.7}$ & 51.9 & 144.3 \\
\hline K00116 & $6.2(K)$ & 7.46 & 2710.6 & $363.2_{-40.3}^{+56.7}$ & 18.1 & 107.1 \\
\hline K00116 & $6.3(K)$ & 13.51 & 4907.8 & $363.2_{-40.3}^{+56.7}$ & 13.2 & 113.8 \\
\hline K00116 & $6.3(K)$ & 13.05 & 4740.8 & $363.2_{-40.3}^{+56.7}$ & 12.7 & 357.4 \\
\hline K00116 & $7.5(K)$ & 10.93 & 3969.3 & $363.2_{-40.3}^{+56.7}$ & 7.5 & 19.7 \\
\hline K00116 & $7.3(K)$ & 5.79 & 2101.4 & $363.2_{-40.3}^{+56.7}$ & 6.3 & 141.1 \\
\hline K00122 & $6.7(J)$ & 4.23 & 1446.2 & $341.7_{-30.3}^{+28.1}$ & 30.0 & 211.3 \\
\hline K00123 & $5.2(J)$ & 9.52 & 4749.5 & $498.7_{-99.9}^{+25.0}$ & 62.3 & 198.8 \\
\hline K00123 & $6.4(J)$ & 10.19 & 5083.7 & $498.7_{-99.9}^{+25.0}$ & 19.2 & 95.2 \\
\hline K00137 & $5.9(J)$ & 5.64 & 2471.4 & $438.2_{-41.0}^{+37.6}$ & 44.4 & 350.7 \\
\hline K00137 & $7.8(J)$ & 7.13 & 3122.2 & $438.2_{-41.0}^{+37.6}$ & 7.6 & 185.5 \\
\hline K00137 & $7.9(J)$ & 5.11 & 2240.3 & $438.2_{-41.0}^{+37.6}$ & 6.6 & 136.2 \\
\hline K00148 & $3.5(J)$ & 4.44 & 1369.1 & $308.7_{-17.2}^{+27.0}$ & 519.8 & 220.6 \\
\hline K00148 & $5.4(J)$ & 10.99 & 3391.9 & $308.7_{-17.2}^{+27.0}$ & 77.0 & 230.1 \\
\hline K00148 & $5.5(J)$ & 2.54 & 785.2 & $308.7_{-17.2}^{+27.0}$ & 69.7 & 245.8 \\
\hline K00148 & $6.3(J)$ & 8.05 & 2486.4 & $308.7_{-17.2}^{+27.0}$ & 35.5 & 244 \\
\hline K00148 & $7.4(J)$ & 6.06 & 1870.7 & $308.7_{-17.2}^{+27.0}$ & 13.9 & 238.8 \\
\hline K00153 & $6.0(K)$ & 8.01 & 1812.0 & $226.2_{-15.1}^{+18.6}$ & 11.2 & 353.4 \\
\hline K00153 & $6.9(J), 7.6(K)$ & 6.17 & 1395.3 & $226.2_{-15.1}^{+18.6}$ & 4.5 & 298.4 \\
\hline K00244 & $2.7(J), 2.0(K)$ & 8.40 & 2741.0 & $326.3_{-44.6}^{+23.4}$ & 3231.4 & 287.6 \\
\hline K00244 & $7.6(J), 7.0(K)$ & 8.38 & 2733.6 & $326.3_{-44.6}^{+23.4}$ & 27.5 & 101.4 \\
\hline K00263 & $1.0(J), 1.0(K)$ & 3.28 & 788.5 & $240.7_{-38.9}^{+17.7}$ & 2430.8 & 268.2 \\
\hline K00273 & $6.2(J), 5.6(K)$ & 5.02 & 1201.8 & $239.6_{-15.2}^{+14.7}$ & 32.6 & 344.0 \\
\hline K00283 & $7.9(K)$ & 6.09 & 1266.2 & $208.0_{-11.2}^{+21.4}$ & 7.0 & 271.4 \\
\hline K00289 & $8.6(J)$ & 5.86 & 2201.2 & $375.9_{-69.8}^{+355.0}$ & 5.1 & 88.3 \\
\hline K00289 & $7.7(J)$ & 3.18 & 1195.0 & $375.9_{-69.8}^{+355.0}$ & 6.1 & 308.6 \\
\hline K00292 & $4.2(K)$ & 0.43 & 154.9 & $358.5_{-28.3}^{+40.9}$ & 43.2 & 119.4 \\
\hline K00365 & $7.7(J), 6.6(K)$ & 7.12 & 1129.9 & $158.8_{-19.7}^{+15.1}$ & 13.3 & 313.7 \\
\hline
\end{tabular}


Table 3

(Continued)

\begin{tabular}{|c|c|c|c|c|c|c|}
\hline \multirow[t]{2}{*}{ KOI } & \multirow{2}{*}{$\begin{array}{l}\Delta \mathrm{Mag} \\
(\mathrm{mag})\end{array}$} & \multicolumn{2}{|c|}{ Separation } & \multirow{2}{*}{$\begin{array}{c}\text { Distance } \\
\text { (pc) }\end{array}$} & \multirow{2}{*}{$\begin{array}{c}\text { Significance } \\
\qquad(\sigma)\end{array}$} & \multirow{2}{*}{$\begin{array}{l}\text { P.A. } \\
\text { (deg) }\end{array}$} \\
\hline & & $(\operatorname{arcsec})$ & $(\mathrm{AU})$ & & & \\
\hline K00377 & $5.0(J), 4.8(K)$ & 6.02 & 3721.9 & $617.9_{-46.7}^{+48.5}$ & 133.8 & 91.9 \\
\hline K00377 & $6.0(J), 6.9(K)$ & 8.04 & 4969.8 & $617.9_{-46.7}^{+48.5}$ & 18.1 & 221.9 \\
\hline K00377 & $6.2(J), 7.3(K)$ & 2.88 & 1780.8 & $617.9_{-46.7}^{+48.5}$ & 12.3 & 37.5 \\
\hline K00975 & $3.8(J), 4.0(K)$ & 0.73 & 90.4 & $123.7_{-17.9}^{+7.7}$ & 31.2 & 133.4 \\
\hline K01442 & $4.7(J)$ & 2.11 & 637.4 & $302.3_{-20.3}^{+18.0}$ & 25.3 & 76.3 \\
\hline K01442 & $8.0(J)$ & 5.69 & 1718.6 & $302.3_{-20.3}^{+18.0}$ & 5.8 & 90.2 \\
\hline
\end{tabular}
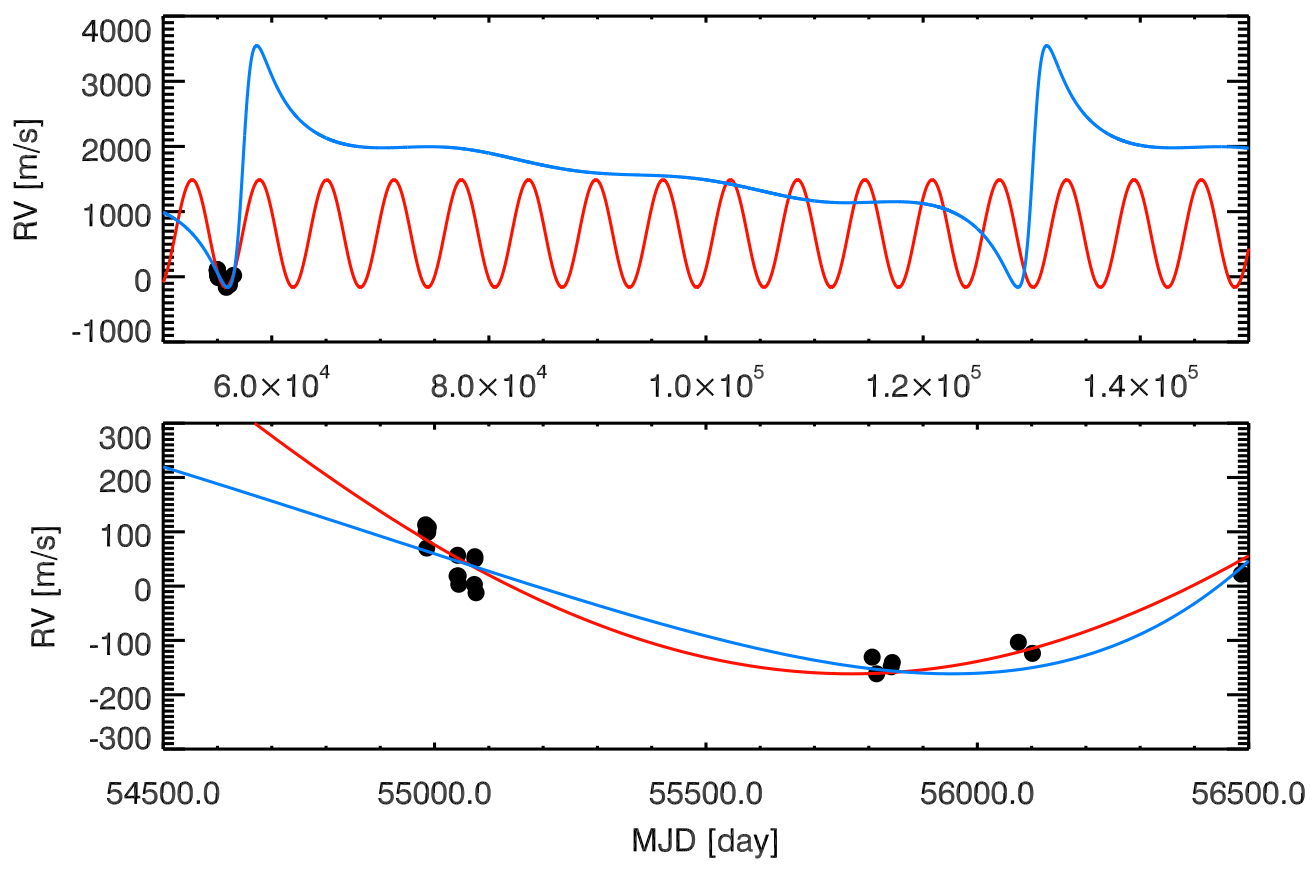

Figure 3. Two possible scenarios for the observed RV acceleration of KOI 5. Black dots are current RV data. Blue line shows a case in which the RV acceleration is

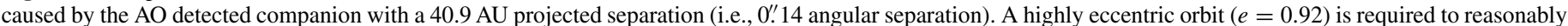
fit the RV data. The red line shows another case in which a $0.08 M_{\odot}$ companion on a circular orbit with a 7 AU separation causes the RV acceleration. More RV data with a longer baseline are required to determine the nature of the companion causing the RV acceleration of KOI 5. The top panel shows a large time range, and the bottom panel shows a zoom-in plot to a time range with RV data.

(A color version of this figure is available in the online journal.)

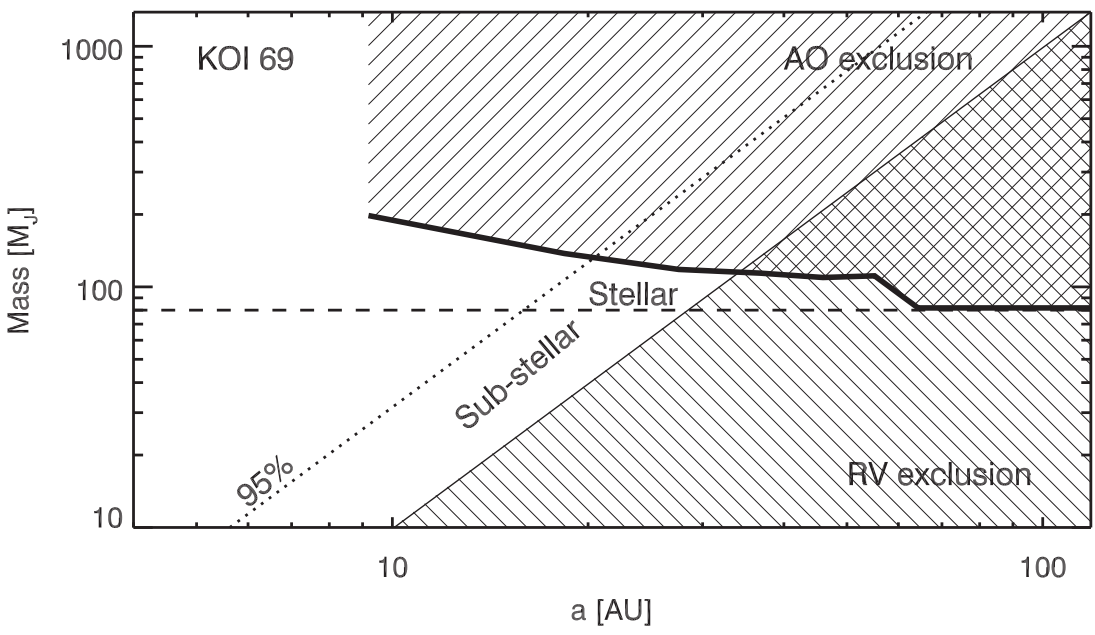

Figure 4. Parameter space for the companion to KOI 69 revealed by an RV linear trend. The region below the solid straight line is excluded because companions falling into this parameter space are not massive and close enough to produce the observed RV linear trend. The solid thick line represents the contrast curve. Any stellar companions above the line would have been detected, so the parameter space above the solid thick line is also excluded. The dashed line divides the stellar regime and substellar regime. The dotted line represents the 95 percentile of the solutions given the linear RV trend, i.e., $95 \%$ of the solutions should fall in between the solid straight line and the dotted line assuming a random orbital orientation of the companion. If the linear RV trend is caused by a stellar companion, then it is most likely the case that the separation is between 15.5 and 33.0 AU, as confined in a parameter space noted with "Stellar." 
host stars. Lillo-Box et al. (2012) estimated that $\sim 35 \%-53 \%$ of visual companions are bonded to the primary stars within $3^{\prime \prime}$, and this ratio decreases with increasing angular separations. Therefore, the nonnegligible fraction of visual companions we detect are in fact unassociated with primary stars, which will decrease the stellar multiplicity rate for planet host stars.

For 12 visual companions with multi-band detections (i.e., $J$ and $K$ band), we test if they are physically associated with their primary stars. The procedures of the test are described as follows. We calculate the $J-K$ colors of visual companions based on the differential magnitudes in Table 3 and the $J-K$ colors of primary stars from the NEA. From their $J-K$ colors, we interpolate for the absolute $K$-band magnitude of companion stars based on Table 5 in Kraus \& Hillenbrand (2007). With the absolute $K$-band magnitudes and the apparent $K$-band magnitudes, we calculate the distances of the visual companion stars, and check whether they are consistent with the distances of the primary stars. If the color-determined distance for the companion is $1 \sigma$ different from the distance of the KOI as reported in Table 3, we reject the physical association between the KOI and the visual companion star. We find inconsistent distance for 6 out of 12 visual companions. The six companions include one for KOI 87 (at 5".49 separation, $d=2.9 \pm 2.2 \mathrm{kpc}$ ), one for KOI 153 (at 6". 17 separation, $d=52 \pm 44 \mathrm{kpc}$ ), one for KOI 244 (at 8."40 separation, $d=33 \pm 20 \mathrm{pc}$ ), and all three for KOI $377(d=3.8 \pm 2.2 \mathrm{kpc}, d=204 \pm 163 \mathrm{kpc}$, and $d=412 \pm 330 \mathrm{kpc})$.

\subsection{Dynamical Analysis}

In addition to constraints from $\mathrm{RV}$ and $\mathrm{AO}$ data, more constraints of potential stellar companions can be put on multiplanet systems (Wang et al. 2014). There are 27 (48\% of the sample) multi-planet systems in our sample for which we can apply the dynamical analysis (DA). The DA technique makes use of the co-planarity of multi-planet systems discovered by the Kepler mission (Lissauer et al. 2011). A stellar companion with high mutual inclination to the planetary orbits would have perturbed the orbits and significantly reduce the co-planarity of planetary orbits, and hence the probability of multi-planet transits. Therefore, the fact that we see multiple planet transiting helps to exclude the possibility of a highly inclined stellar companion. The DA is complementary to the RV technique because it is sensitive to stellar companions with large mutual inclinations to the planetary orbits. The parameter space the DA is sensitive to is shown in Figure 1.

\subsection{Combining Results from Different Techniques}

For the RV and AO observations, detection completeness contours are calculated based on simulations given the observational constraints, such as the time baseline, cadence, measurement uncertainties, and the contrast curve. For the DA technique, numerical integrations give the fraction of time when multiple planets can stay with small mutual inclinations $\left(<5^{\circ}\right)$ so that multiple transiting planets can be observed (Wang et al. 2014). Note that the DA technique works only for systems with multiple planets, which account for $48 \%$ of the sample. For systems with a single transiting planet, no constraint can be given by the DA technique. We denote $c_{\mathrm{RV}}, c_{\mathrm{AO}}$, and $c_{\mathrm{DA}}$ as the completenesses at a given point in the $a-i$ parameter space, overall completeness $c$ is equal to $1-\left(1-c_{\mathrm{RV}}\right) \times\left(1-c_{\mathrm{AO}}\right) \times\left(1-c_{\mathrm{DA}}\right)$. We note that the calculation assumes each technique is independent and uniquely sensitive to a certain portion of the parameter space. This is generally the case since the RV technique completeness drops quickly beyond $\sim 30 \mathrm{AU}$, where the $\mathrm{AO}$ technique sensitivity is high. Similarly, the RV and DA techniques and the DA and AO techniques have little overlap in sensitivity parameter space. The overall completeness may be overestimated at the transition space, such as $a=30 \mathrm{AU}$ (for $\mathrm{RV}$ and $\mathrm{AO}$ ) and $i=20^{\circ}$ (for $\mathrm{RV}$ and DA), because stellar companions falling into this parameter space can be detected by multiple techniques and thus the techniques become correlated. We also try another way of combining results from different techniques, in which we use the maximum completeness as the overall completeness. This approach assumes multiple techniques are correlated, however, it does not significantly change the conclusions in this paper.

The completeness is then integrated over the $a-i$ parameter space. We assume a log-normal distribution for $a$ (Duquennoy \& Mayor 1991; Raghavan et al. 2010), random distribution of $i$ for systems with only one transiting planet, and the $i$ distribution from Hale (1994) for systems with multiple transiting planets. The treatment for multiple transiting planet systems is detailed in Wang et al. (2014), i.e., a coplanar distribution for stellar companions within $15 \mathrm{AU}$, a random $i$ distribution for stellar companions beyond $30 \mathrm{AU}$, and a mixture $i$ distribution at intermediate separations between 15 and $30 \mathrm{AU}$.

\section{PLANET OCCURRENCE RATE AND STELLAR MULTIPLICITY RATE}

\subsection{Detection Bias Against Planets in Multiple-star Systems}

Planets in multiple-star systems are more difficult to find using the transiting method because of flux contamination. We discuss how this bias against planet detection in multiplestar systems can be quantified. For the Kepler mission, it is a necessary condition to become a planet candidate that the signalto-noise ratio $(\mathrm{S} / \mathrm{N})$ should be higher than 7.1 (Jenkins et al. 2010a). S/N can be calculated using the following equation:

$$
\mathrm{S} / \mathrm{N}=\frac{\delta}{\mathrm{CDPP}_{\text {eff }}} \sqrt{N_{\text {transits }}},
$$

where $\delta$ is the transit depth, $\mathrm{CDPP}_{\text {eff }}$ is the effective combined differential photometric precision (Jenkins et al. 2010b), a measure of photometric noise, and $N_{\text {transits }}$ is the number of observed transits. We use a planet in a binary system as an example to calculate the transit depth:

$$
\delta=\frac{R_{\mathrm{PL}}^{2}}{R_{*}^{2}} \frac{F_{*}}{F_{*}+F_{c}},
$$

where $R_{\mathrm{PL}}$ is planet radius, $R_{*}$ is the radius of the star that the planet is transiting, $F$ denotes flux, and subscript $*$ and $c$ indicate the planet host star and the contaminating star, respectively. Two cases are considered for the above equation. First, if the planet transits the primary star, the transit depth is diluted by a factor of two at most, when $F_{*}$ and $F_{c}$ are identical. Second, if the planet transits the secondary star, the transit depth dilution effect due to flux contamination can be much larger than two even after considering the increase in the transit depth from a reducing $R_{*}$ in the first term of the equation. For an example of a solar-type star and a late-type $\mathrm{M}$ dwarf pair, the gain of a reducing $R_{*}$ can be a factor of 100 at most, but the flux ratio between the two stars can easily exceed $10^{4}$ in the Kepler band.

Therefore, we conduct simulations to quantify the detection bias against planets in binary star systems. For each KOI, we 
choose the one planet that gives the highest $\mathrm{S} / \mathrm{N}$. We add a companion star in the system and calculate the $\mathrm{S} / \mathrm{N}$ in the presence of flux contamination for two cases: planet transiting the primary star and planet transiting the secondary star. In both cases, we assume the same period and transit duration from the NEA so that $C D P P_{\text {eff }}$ and $N_{\text {transits }}$ in Equation (1) are the same, and flux contamination (see Equation (2)) is the only factor that determines whether a planet is detected in the presence of a companion star. If the $\mathrm{S} / \mathrm{N}$ is higher than 7.1, then the planet can still be detected by Kepler, but with a lower significance. We randomly assign a stellar companion (secondary star) to a KOI (primary star) and repeat this procedure 1000 times for both the primary and the secondary star. We record the fraction of planet detections, $\alpha$, which will be used in correcting for the bias of detecting planets in multiple-star systems (Table 4). The median value of $\alpha$ for 56 stars in our sample is 0.89 , implying that the detection bias is not severe, but certainly not negligible.

In the simulations, we use the stellar parameters from the NEA for the primary star. When generating a stellar companion in the simulations, we assume the mass ratio distribution follows the normal distribution given in Duquennoy \& Mayor (1991). The radius of the secondary star is calculated using a stellar mass-radius relationship (Feiden \& Chaboyer 2012). Estimation of stellar flux for both primary and secondary stars are based on Table 5 in Kraus \& Hillenbrand (2007). We calculated $C D P P_{\text {eff }}$ by interpolating between 3, 6, and $12 \mathrm{hr}$ CDPPs based on transit duration.

\subsection{Distinguishing Planets in Single and Multiple-star Systems}

The Kepler mission has provided us with a large sample of planet candidates. However, we do not know whether the planet host stars are in single or multiple stellar systems. Distinguishing planets in single and multiple-star systems allows us to separately calculate the planet occurrence rate for these two types of stars, and to understand planet formation in different stellar environments (Wang et al. 2014). Follow-up observations are critical in identifying additional stellar companions in planetary systems. Even in the case of nondetection, with RV, AO, and DA techniques, we can calculate the probability of a star being in a multiple-star system based on the completeness study. For example, if the overall completeness for a companion detection is $80 \%$ and the stellar multiplicity rate is $46 \%$ (Raghavan et al. 2010), then the probability of the star having an undetected companion (or being a multiple-star) is $(100 \%-80 \%) \times 46 \%=$ 0.092 . Following this procedure, we calculate the number of multiple-stars $N_{M}$ and the number of single stars $N_{S}$. Since $N_{M}$ and $N_{S}$ are the sums of probabilities, they will not necessarily be integers:

$$
N_{M}=\sum_{i=1}^{n}\left[p_{M}(i) / \alpha(i)\right], \quad N_{S}=\sum_{i=1}^{n}\left[1-p_{M}(i)\right],
$$

where $n$ is the total number of stars in the sample, $p_{M}(i)$ is the probability of the $i_{\text {th }}$ star being a multiple-star system, $\alpha(i)$ is the correction factor for the detection bias for planets in multiplestar systems. The above equation is similar to Equation (6) in Wang et al. (2014) except for the correction factor $\alpha$. Note that there is an implicit correction factor for single stars in Equation (3). However, the correction factor for single stars is one. If a stellar companion is detected for a KOI, then $p_{M}$ is assigned to one, and $\alpha$ is also assigned to one because no bias exists in this case since a planet has already been detected in a multiple-star system. For an AO detected stellar companion, setting $p_{M}$ to one is an overestimation because the physical association of visual stellar components is not yet established. Therefore, the stellar multiplicity rate that will be subsequently determined is an upper limit.

We then define $f$ as the fraction of stars with planets, $f$ can be separated into two components:

$$
f=(1-\mathrm{MR}) \times f_{S}+\mathrm{MR} \times f_{M},
$$

where MR is the global stellar multiplicity rate, $f_{S}$ and $f_{M}$ are the fraction of stars with planets for single and multiple-star systems, respectively. The ratio of $f_{S}$ and $f_{M}$ can be calculated in the following equation:

$$
\frac{f_{S}}{f_{M}}=\frac{\frac{N_{S}}{1-\mathrm{MR}}}{\frac{N_{M}}{\mathrm{MR}}}
$$

With Equations (4) and (5), $f_{S}$ and $f_{M}$ can be solved independently given that $N_{S}$ and $N_{M}$ can be measured and that $f$ can be measured globally (e.g., Fressin et al. 2013). In addition, the MR for planet host stars $\left(\mathrm{MR}_{\mathrm{PL}}\right)$ can be calculated and compared to a global MR:

$$
\mathrm{MR}_{\mathrm{PL}}=\frac{N_{M}}{N_{M}+N_{S}},
$$

\subsection{Stellar Multiplicity Rate for Planet Host Stars}

Figure 5 shows the comparison between the stellar multiplicity rate for field stars (dashed line Duquennoy \& Mayor 1991; Raghavan et al. 2010) and that for planet host stars (blue and red hatched regions). The red hatched region is the $1 \sigma$ uncertainty region for 56 stars with $\mathrm{RV}$ and $\mathrm{AO}$ observations, and the DA analysis. The error bar of $N_{M}$ is estimated based on Poisson statistics. The square root of the closest integer to $N_{M}$ is used as the error bar to $N_{M}$ unless the closest integer is zero, in which case we used one for the error of $N_{M}$. The stellar multiplicity rate for planet host stars is significantly lower than that of field stars until the separation reaches $\sim 1500$ AU. This implies that the influence of a stellar companion may be more profound than previously thought. The effective separation below which planet formation is significantly affected is extended to $\sim 1500 \mathrm{AU}$. In comparison, the blue hatched region represents the $1 \sigma$ uncertainty region for 23 stars with RV data and DA analysis, but no AO observations (Wang et al. 2014). Based on the blue hatched region, the significant difference of stellar multiplicity disappears after separation reaches 20.8 AU. Since Wang et al. (2014), we have incorporated AO data into our analyses and increased the sample size from 23 to 56. These improvements greatly strengthen the statistics in the comparison. Specifically, increasing the sample size reduces the statistical uncertainty; adding AO data helps constrain stellar companions beyond the reach of the RV technique.

\subsection{Planet Occurrence Rate versus Binary Separation}

With the stellar multiplicity rate for planet host stars, we can calculate the ratio of the planet occurrence rate for single and multiple-star systems according to Equation (5). Figure 6 shows the ratio $f_{S} / f_{M}$ as a function separation. Planets orbiting single stars are $4.5 \pm 3.2,2.6 \pm 1.0$, and $1.7 \pm 0.5$ times more likely than planets in S-type orbits in multiple-star systems with stellar separations of $10 \mathrm{AU}, 100 \mathrm{AU}$, and $1000 \mathrm{AU}$, respectively. 
Table 4

Detection Bias of Planets in Multiple Stars

\begin{tabular}{|c|c|c|c|c|c|c|c|c|}
\hline KOI & $\begin{array}{l}\text { Period } \\
\text { (day) }\end{array}$ & $\begin{array}{c}R_{P} \\
\left(R_{\oplus}\right) \\
\end{array}$ & $\begin{array}{c}R_{*} \\
\left(R_{\odot}\right)\end{array}$ & $\begin{array}{c}M_{*} \\
\left(M_{\odot}\right)\end{array}$ & $\begin{array}{l}\text { Duration } \\
\text { (hr) }\end{array}$ & $\begin{array}{c}\mathrm{CDPP}_{\mathrm{eff}}^{\mathrm{a}} \\
(\mathrm{ppm})\end{array}$ & Quarters & $\alpha^{\mathrm{b}}$ \\
\hline K00005 & 4.78033 & 5.66 & 1.42 & 1.15 & 2.01 & 36.3 & 17 & 0.930 \\
\hline K00007 & 3.21366 & 3.72 & 1.27 & 1.12 & 4.11 & 53.1 & 14 & 0.850 \\
\hline K00010 & 3.52250 & 15.90 & 1.56 & 1.14 & 3.20 & 136.0 & 17 & 0.961 \\
\hline K00017 & 3.23470 & 11.07 & 1.08 & 1.14 & 3.60 & 103.5 & 14 & 0.946 \\
\hline K00018 & 3.54847 & 17.40 & 2.02 & 1.45 & 4.08 & 102.7 & 17 & 0.619 \\
\hline K00020 & 4.43796 & 17.60 & 1.38 & 1.17 & 4.67 & 106.1 & 14 & 0.961 \\
\hline K00022 & 7.89145 & 11.27 & 1.11 & 1.16 & 3.79 & 82.4 & 17 & 0.938 \\
\hline K00041 & 12.81570 & 2.08 & 1.23 & 1.11 & 6.54 & 26.6 & 17 & 0.635 \\
\hline K00069 & 4.72675 & 1.50 & 0.87 & 0.89 & 2.93 & 17.9 & 17 & 0.938 \\
\hline K00070 & 10.85410 & 3.17 & 0.94 & 0.90 & 3.82 & 57.1 & 17 & 0.930 \\
\hline K00072 & 0.83749 & 1.37 & 1.00 & 0.91 & 1.80 & 29.6 & 14 & 0.938 \\
\hline K00082 & 16.14570 & 2.54 & 0.74 & 0.80 & 3.75 & 39.4 & 17 & 0.946 \\
\hline K00084 & 9.28701 & 2.53 & 0.86 & 0.91 & 3.54 & 34.3 & 17 & 0.938 \\
\hline K00085 & 5.85993 & 2.36 & 1.20 & 1.21 & 4.11 & 29.0 & 17 & 0.624 \\
\hline K00087 & 289.86200 & 2.10 & 0.85 & 0.83 & 7.40 & 23.4 & 17 & 0.773 \\
\hline K00103 & 14.91080 & 2.95 & 0.95 & 0.91 & 3.31 & 73.7 & 17 & 0.906 \\
\hline K00104 & 2.50806 & 3.36 & 0.76 & 0.81 & 1.14 & 84.9 & 17 & 0.961 \\
\hline K00108 & 15.96530 & 2.94 & 1.21 & 1.16 & 4.65 & 32.2 & 17 & 0.629 \\
\hline K00111 & 11.42750 & 2.14 & 0.93 & 0.81 & 4.59 & 47.5 & 17 & 0.930 \\
\hline K00116 & 13.57070 & 2.47 & 1.04 & 1.00 & 3.25 & 59.2 & 17 & 0.803 \\
\hline K00122 & 11.52310 & 2.78 & 1.09 & 1.07 & 4.06 & 45.2 & 17 & 0.773 \\
\hline K00123 & 6.48167 & 2.64 & 1.43 & 1.06 & 3.63 & 40.9 & 17 & 0.874 \\
\hline K00137 & 14.85890 & 6.01 & 0.98 & 0.94 & 3.63 & 83.8 & 17 & 0.954 \\
\hline K00148 & 9.67393 & 3.15 & 0.89 & 0.88 & 4.40 & 72.8 & 17 & 0.930 \\
\hline K00153 & 8.92511 & 2.47 & 0.69 & 0.74 & 2.77 & 91.0 & 17 & 0.938 \\
\hline K00157 & 31.99550 & 4.18 & 1.06 & 0.98 & 4.27 & 77.1 & 17 & 0.906 \\
\hline K00180 & 10.04560 & 2.53 & 0.92 & 0.99 & 3.26 & 62.0 & 17 & 0.866 \\
\hline K00244 & 12.72040 & 6.51 & 1.66 & 1.19 & 2.83 & 28.4 & 17 & 0.898 \\
\hline K00245 & 39.79220 & 1.94 & 0.73 & 0.75 & 4.57 & 17.3 & 17 & 0.946 \\
\hline K00246 & 5.39877 & 2.53 & 1.24 & 1.07 & 3.56 & 22.0 & 17 & 0.922 \\
\hline K00261 & 16.23850 & 2.65 & 1.02 & 0.99 & 3.86 & 36.0 & 17 & 0.914 \\
\hline K00263 & 20.71940 & 2.02 & 1.41 & 1.01 & 4.23 & 49.2 & 17 & 0.658 \\
\hline K00265 & 3.56806 & 1.29 & 1.18 & 1.16 & 3.43 & 36.1 & 17 & 0.528 \\
\hline K00273 & 10.57380 & 1.82 & 1.07 & 1.12 & 1.74 & 30.4 & 17 & 0.624 \\
\hline K00274 & 15.09200 & 1.13 & 1.55 & 1.20 & 4.14 & 28.0 & 17 & 0.500 \\
\hline K00283 & 16.09190 & 2.41 & 1.03 & 1.02 & 2.93 & 31.4 & 17 & 0.874 \\
\hline K00289 & 296.63700 & 5.04 & 0.95 & 0.94 & 16.43 & 28.3 & 17 & 0.930 \\
\hline K00292 & 2.58663 & 1.64 & 0.98 & 0.94 & 2.37 & 53.9 & 14 & 0.874 \\
\hline K00299 & 1.54168 & 1.98 & 1.11 & 0.99 & 1.94 & 84.7 & 17 & 0.890 \\
\hline K00305 & 4.60356 & 1.57 & 0.73 & 0.79 & 2.40 & 74.9 & 17 & 0.898 \\
\hline K00321 & 2.42631 & 1.50 & 1.11 & 0.99 & 2.65 & 50.7 & 17 & 0.850 \\
\hline K00364 & 173.92800 & 0.93 & 1.35 & 1.15 & 2.64 & 23.0 & 17 & 0.500 \\
\hline K00365 & 81.73750 & 2.29 & 0.87 & 0.85 & 6.78 & 23.7 & 17 & 0.906 \\
\hline K00377 & 19.27390 & 8.28 & 1.01 & 1.05 & 4.16 & 129.3 & 17 & 0.930 \\
\hline K00701 & 18.16410 & 1.91 & 0.60 & 0.65 & 2.96 & 83.3 & 17 & 0.922 \\
\hline K00975 & 2.78582 & 1.72 & 2.04 & 1.36 & 3.41 & 24.1 & 17 & 0.500 \\
\hline K01431 & 345.16100 & 8.45 & 1.00 & 1.06 & 7.50 & 45.8 & 14 & 0.890 \\
\hline K01439 & 394.61100 & 7.80 & 1.65 & 1.23 & 24.61 & 15.3 & 17 & 0.635 \\
\hline K01442 & 0.66934 & 1.23 & 0.99 & 1.01 & 1.29 & 54.7 & 14 & 0.795 \\
\hline K01463 & 580.00000 & 16.29 & 1.09 & 1.05 & 11.43 & 38.6 & 17 & 0.961 \\
\hline K01612 & 2.46503 & 0.78 & 1.31 & 1.05 & 1.19 & 16.7 & 14 & 0.629 \\
\hline K01781 & 7.83445 & 3.76 & 0.76 & 0.82 & 3.00 & 80.5 & 14 & 0.946 \\
\hline K01925 & 68.95800 & 1.12 & 0.95 & 0.88 & 2.99 & 15.4 & 17 & 0.658 \\
\hline K02169 & 5.45300 & 0.97 & 0.93 & 0.82 & 2.24 & 43.2 & 17 & 0.723 \\
\hline K02687 & 1.71683 & 1.90 & 1.94 & 1.12 & 2.11 & 24.2 & 17 & 0.818 \\
\hline K02720 & 6.57148 & 0.80 & 1.05 & 1.05 & 3.07 & 22.6 & 17 & 0.619 \\
\hline
\end{tabular}

Notes.

${ }^{a}$ Effective combined differential photometric precision (Jenkins et al. 2010b).

${ }^{\mathrm{b}}$ Correction factor for the bias against planet detection in binary stars. The factor ranges from zero to one, with one indicating $100 \%$ detection rate even with the flux contamination from a companion star. See Section 4.1 for more details. 


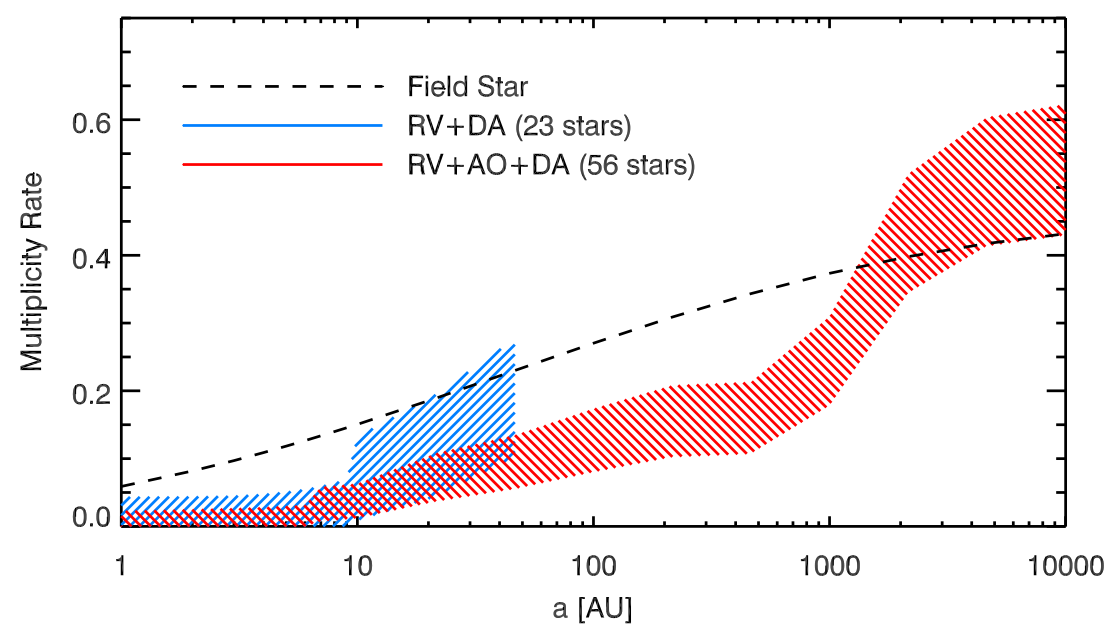

Figure 5. Comparison of the stellar multiplicity rate of field stars (dashed line) and planet host stars (hatched regions). The blue hatched region represents the $1 \sigma$ region of the stellar multiplicity rate for 23 planet host stars with RV and DA analysis (Wang et al. 2014). AO data were not incorporated, so the sensitivity of RV and DA was limited within $50 \mathrm{AU}$. For this study, AO data are used to constrain stellar companions beyond $50 \mathrm{AU}$. The red hatched region represents the $1 \sigma$ region of the stellar multiplicity rate for 56 stars with RV, AO, and DA analysis. The new study shows that the stellar multiplicity rate for planet host stars is lower than that for the field stars within $1500 \mathrm{AU}$, indicating a more profound influence of stellar companions on planet formation.

(A color version of this figure is available in the online journal.)

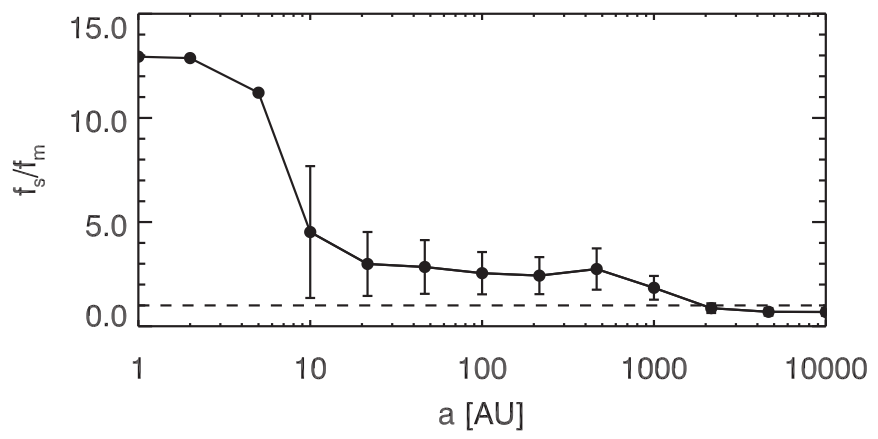

Figure 6. Ratio of the planet occurrence rates for single and multiple-stars. Dashed line represents the value of one, a value indicating a comparable planet occurrence rate. The planet occurrence rate for single stars is much higher than that for multiple-stars within $10 \mathrm{AU}$. Beyond $10 \mathrm{AU}$, the ratios are $4.5 \pm 3.2$, $2.6 \pm 1.0$, and $1.7 \pm 0.5$ for $10 \mathrm{AU}, 100 \mathrm{AU}$, and $1000 \mathrm{AU}$, respectively, indicating planets in multiple-star systems are fewer than those around single stars at these separations. The planet occurrence rates become comparable between single and multiple stars when separation is larger than $\sim 1500 \mathrm{AU}$. Error bars are calculated based on Poisson statistics and propagated through Equation (5). No error bar is shown within $10 \mathrm{AU}$ because of the detection of less than one stellar companion according to Equation (3).

The deficiency of planets around multiple-stars indicates that the suppressive influence on planet formation of a stellar companion is significant at these separations. The suppressive effect decreases as separation increases, and $f_{S}$ and $f_{M}$ are comparable at separations around $\sim 1500 \mathrm{AU}$, indicating that stellar companions at these separations barely have any influence on planet formation. The comparison of planet occurrence rate for single and multiple-stars at other stellar separations is given in Table 5.

\subsection{Comparison to Previous Results}

The field of studying planets in multiple-star systems may be divided into two eras: before and after the Kepler mission. Before the Kepler mission, stars with giant planets are the main targets, and they are mostly detected by the RV technique. Bonavita \& Desidera (2007) used a sample defined as the "uniform detectability" (UD) sample. They searched for stellar companions around stars in this sample, and found that the
Table 5

Ratio of the Planet Occurrence Rate Between Single Stars and Multiple-Star Systems as a Function of Stellar Separation

\begin{tabular}{rrr}
\hline \hline \multicolumn{1}{l}{$\begin{array}{l}\text { (AU) } \\
1.0\end{array}$} & $f_{s} / f_{m}$ & $\delta f_{s} / f_{m}{ }^{a}$ \\
2.0 & 12.94 & \\
5.0 & 12.87 & $\ldots$ \\
10.0 & 11.21 & $\ldots$ \\
21.5 & 4.52 & 3.16 \\
46.4 & 2.99 & 1.53 \\
100.0 & 2.84 & 1.29 \\
215.4 & 2.55 & 1.01 \\
464.2 & 2.43 & 0.89 \\
1000.0 & 2.75 & 0.99 \\
2154.4 & 1.84 & 0.57 \\
4641.6 & 0.87 & 0.23 \\
10000.0 & 0.69 & 0.18 \\
\hline
\end{tabular}

Notes. ${ }^{\text {a }}$ Error bars are calculated based on Poisson statistics and propagated through Equation (5). No error bar is given within 10 AU because of the detection of less than one stellar companion according to Equation (3).

fractions of stars with detected planets are comparable between single and multiple-stars. However, after considering the search incompleteness, they concluded that the frequency of planets in binary stars cannot be more than a factor of three lower than that of single stars. Their finding is consistent with our conclusion for separations larger than $\sim 50 \mathrm{AU}$. However, we find that $f_{s} / f_{m}$ can be higher than three for shorter binary separations (Table 5). Eggenberger et al. (2011) presented comparison of stellar multiplicity rate between planet host stars and a control sample of nonplanet host stars. They concluded that S-type gas giant planets are less frequent in binary stars with mean semi-major axes between 35 and $250 \mathrm{AU}$. Their conclusion is qualitatively consistent with ours, but we find that planet formation can be suppressed at larger separations (out to $1500 \mathrm{AU})$. We emphasize that there are fundamental differences in the comparison to previous results on RV planet surveys. First, they focused on host stars of gas giant planets, whereas this study 


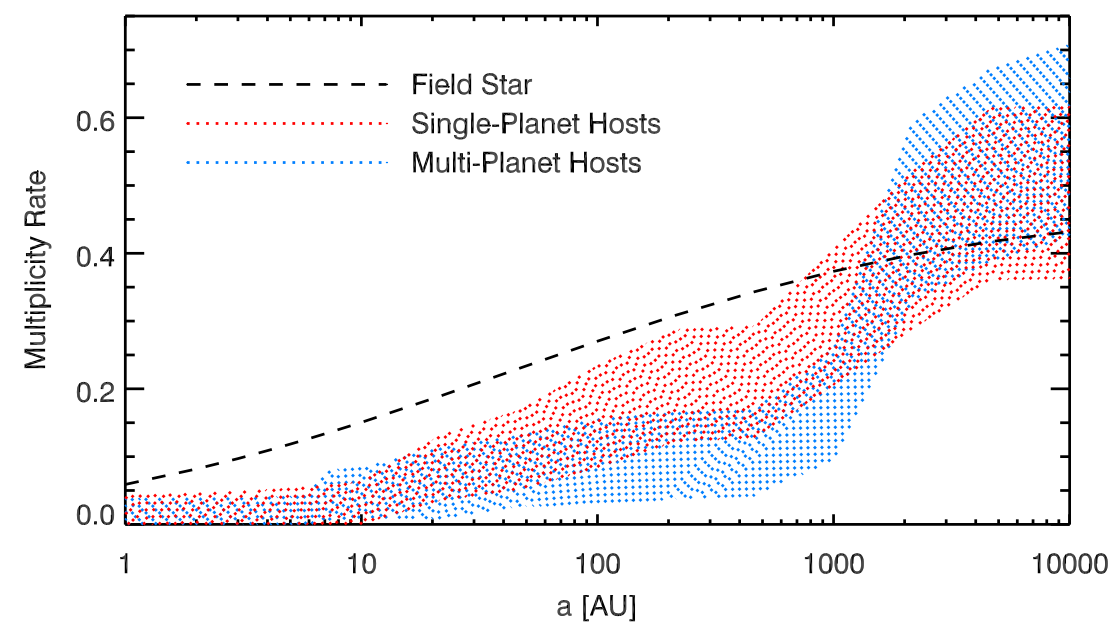

Figure 7. Comparison of the stellar multiplicity rate for field stars (dashed line), 29 planet host stars with a single detected planet (red dotted region, $1 \sigma$ range), and 27 planet host stars with multiple detected planets (blue dotted region, $1 \sigma$ range).

(A color version of this figure is available in the online journal.)

has made use of Kepler data, and therefore mostly deals with lower-mass planets. Second, RV surveys have a much stronger bias against close-in binary stars than the Kepler mission.

After the Kepler satellite was launched, studies continue on the stellar multiplicity of planet host stars. Lillo-Box et al. (2012) found that the visual companion rate for KOIs is $17.3 \%$ and $41.8 \%$ within $3^{\prime \prime}$ and 6 ", respectively. They later updated the companion rate to be $17.2 \%$ and $32.8 \%$ within $3^{\prime \prime}$ and $6^{\prime \prime}$ (LilloBox et al. 2014). Dressing et al. (2014) found that $17.2 \%$ of KOIs have visual companions within $3^{\prime \prime}$. In comparison, we find that $12.5 \pm 4.7 \%$ and $48.2 \pm 9.3 \%$ of KOIs have visual companions within $3^{\prime \prime}$ and 6 ", which is consistent with their numbers. Adams et al. (2012) found that $60 \%, 20 \%$, and $7 \%$ of 90 KOIs have stellar companions within $6^{\prime \prime}, 2^{\prime \prime}$, and 0.5 , respectively. We find that these numbers to be $48.2 \pm 9.3 \%$, $5.4 \pm 3.1 \%$, and $3.6 \pm 2.5 \%$. In comparison, we find significantly fewer stellar companions than Adams et al. (2012) at angular separations between 0.5 and $2 . \prime 0$.

We therefore conduct a cross-check with their targets, and find 20 overlapping targets. For these targets, we detect 40 companions using the images from the CFOP, while they detect 33 companions. We find 17 new companions that were not reported by Adams et al. (2012). Most of the new companions are more than $6^{\prime \prime}$ away from central stars. We are not able to detect 10 of their companions. All of our missing detections have $\Delta$ Mag larger than $7.1 \mathrm{mag}$ (close to detection limit, see Figure 2), and none of them are within $2^{\prime \prime}$ except for KOI 18 $\left(0^{\prime \prime} 9\right.$ separation and $\left.\Delta \mathrm{mag}=5.0\right)$. We suspect the difference may be a result of different thresholds for companion detections or differences in manual inspections.

We also conduct investigations on the lack of companion detections within 2 ". 0 . In the overlapping sample of 20 KOIs with Adams et al. (2012), we detect two companions within 0.5, KOI 292 (0'.43), and KOI 975 (0'72). They are also detected in Adams et al. (2012), but KOI 18 with a separation of 0!9 was missed in our search. For the overlapping sample, 10.0 $7.1 \%$ (2 out 20) have companions within $2^{\prime \prime}$. In comparison, for the rest of our sample, none of the 36 stars have companions detected within $2^{\prime \prime}$, which raises a concern that KOIs with close-in companions may be filtered out when conducting RV followup observations. However, it does not seem to be the case for KOI 18, KOI 292, and KOI 975, these targets receive continued
RV followup observations even after close-in companions are detected in $\mathrm{AO}$ images.

\section{SUMMARY AND DISCUSSION}

\subsection{Summary}

We conduct a search for stellar companions to a sample of 56 Kepler planet host stars, and compare the stellar multiplicity rate for planet host stars and the field stars in the solar neighborhood. We find that the stellar multiplicity rate for planet host stars is significantly lower than that for the field stars at stellar separations smaller than $1500 \mathrm{AU}$, indicating that planet formation is less efficient in multiple-star systems than in single stars. The influence of stellar companions plays a significant role in planet formation and evolution in multiple-star systems with separations smaller than $1500 \mathrm{AU}$.

We distinguish the planet occurrence rates for single and multiple-stars. We find that planets in S-type orbits in multiplestar systems are $4.5 \pm 3.2,2.6 \pm 1.0$, and $1.7 \pm 0.5$ times less frequent than planets orbiting single stars if a stellar companion is present at distances of 10,100, and $1000 \mathrm{AU}$, respectively. The difference in planet occurrence rate between single and multiple-star systems becomes insignificant when companion separation exceeds $1500 \mathrm{AU}$, suggesting that planet formation in widely separated binaries is similar to that around single stars.

In summary, three improvements in this study allow us to better study planets in multiple-star systems. First, unlike planet host stars selected from ground-based RV and transiting surveys, our sample from the Kepler mission does not have strong bias against planets in multiple-star systems. Second, we combine the RV and AO data for the 56 Kepler stars, which construct a survey for stellar companions with high completeness. The DA method is also used to put further constraints on stellar companions in systems with multiple transiting planets. Third, we develop a method to quantify the detection bias of planets in multiple-star systems, which enables a fair comparison of stellar multiplicity rate.

\subsection{Discussion}

\subsubsection{Stellar Companions to Hot Jupiter Host Stars}

There are six hot Jupiter (HJ, $P<10$ day and $\left.R_{P}>5 R_{\oplus}\right)$ host stars in our sample. They are KOI 5, KOI 10, KOI 17, 
KOI 18 , KOI 20, and KOI 22. Four $(67 \%)$ of them have detected stellar companions. The stellar multiplicity rate for $\mathrm{HJ}$ host stars is much higher than the rest of the sample, i.e., $32 \%$. While we recognize the small number statistics and the possible nonassociation of these visual companions, this may imply that stellar companions play a role in HJ migration. Knutson et al. (2014) conducted a search for massive companions to close-in gas giant planets. They estimated an occurrence rate of $51 \% \pm 10 \%$ for companions with masses between 1 and $13 M_{J}$ and semi-major axes between 1 and $20 \mathrm{AU}$. The high occurrence rate for both massive sub-stellar companions and stellar companions may suggest that planet-planet and star-planet interactions have a comparable influence on the migration of HJs. Given the large separations of stellar companions $(a>1500 \mathrm{AU})$, the Kozai timescales for all $\mathrm{HJ}$ systems with stellar companions (except for KOI 5) are $\sim 10^{8}-10^{9} \mathrm{yr}$, which are comparable to the age of the systems, and perhaps too long to effectively perturb the orbit of a gas giant planet. Therefore, it is still inconclusive whether the HJs in these systems migrate to their current positions due the perturbation of the detected stellar companions.

\subsubsection{Stellar Multiplicity Rate for Single and Multiple Planet Systems}

Perturbation from a companion star will change the mutual inclinations of planets in the same system (Wang et al. 2014, see also Section 3.3). We therefore expect to see a lower stellar multiplicity rate for stars with multiple transiting planets than stars with only one transiting planet. There are 27 stars in our sample with multiple transiting planets and 29 stars with only one transiting planet. Figure 7 shows the comparison of stellar multiplicity rate for these two sub samples. The hatched regions with different colors overlap, so there is no statistically significant difference in the stellar multiplicity rate between systems with multiple transiting planets and systems with only one transiting planet. However, for separations between 50 and $1000 \mathrm{AU}$, we notice a relatively lower stellar multiplicity rate for multiple transiting planet systems than systems with only one detected transiting planet, suggesting that companion perturbations affect planet mutual inclination and/or multiple planet formation. An ongoing AO campaign is being carried out at the Palomar observatory to study the stellar multiplicity rate for multi-planet host stars, and will address the role of stellar perturbation in planet formation and detection.

\subsubsection{Needing a Better Control Sample than the Field Stars}

There are several uncertainties when using the field stars as a control sample to compare to the Kepler sample. First, it is uncertain that Kepler's overall sample (i.e., all Kepler stars) multiplicity rate is the same as that of the field stars. Kepler stars are mainly selected by applying a magnitude cut (magnitudelimited), whereas the field stars are volume-limited (Duquennoy \& Mayor 1991; Raghavan et al. 2010). Therefore, Malmquist bias exists for the Kepler stars, brighter, more distant stars, are selected, which include young stars, giant stars, and binary stars. While some young stars and giant stars can be excluded by applying a $T_{\text {eff }}$ and $\log (g)$ cut from the Kepler Input Catalog (KIC; Brown et al. 2011), it is more difficult to discern binary stars, so it is possible that the stellar multiplicity rate for Kepler stars could be higher than that of the field stars (Gaidos \& Mann 2013).

The second uncertainty lies in the fraction of field stars with planets. We compare the stellar multiplicity rate for the field stars and planet host stars. Most of them are small planet host stars. However, the fraction of field stars hosting small planets is less constrained than the fraction of field stars hosting large planets (Howard et al. 2010). If (1) not all field stars have a planet; and (2) the statistics of multiple-stars (multiplicity, separation distribution, etc.) are comparable for the nearby solartype stars and for the stars in our sample, then the difference in Figure 5 should suggest the impact of stellar companions on planet occurrence. In this case, the field stars are a sample contaminated by planet host stars. If we see a difference when comparing a sample of planet host stars to the field stars, then the difference would have been more distinct when comparing the planet host sample and a nonplanet host sample. The latter is difficult to obtain because of the limitation of current detection sensitivity. However, a planet mass or radius limit can be set to study a certain type of planet, e.g., comparing the stellar multiplicity rate for the giant planet host stars and stars without a gas giant planet. In this case, any difference in the multiplicity rate reflects the impact of stellar companions on gas giant planet formation.

The authors thank Howard Isaacson and Matt Giguere for helpful comments and proofreading the paper. The research is made possible by the data from the Kepler Community Followup Observing Program (CFOP). The authors acknowledge all the CFOP users who uploaded the AO and RV data used in the paper. This research has made use of the NASA Exoplanet Archive, which is operated by the California Institute of Technology, under contract with the National Aeronautics and Space Administration under the Exoplanet Exploration Program. Jiwei Xie is supported by a Foundation for the Author of National Excellent Doctoral Dissertation (FANEDD) of PR China.

\section{REFERENCES}

Adams, E. R., Ciardi, D. R., Dupree, A. K., et al. 2012, AJ, 144, 42 Adams, E. R., Dupree, A. K., Kulesa, C., \& McCarthy, D. 2013, AJ, 146, 9 Batalha, N. M., Rowe, J. F., Bryson, S. T., et al. 2013, ApJS, 204, 24 Bergfors, C., Brandner, W., Daemgen, S., et al. 2013, MNRAS, 428, 182 Bonavita, M., \& Desidera, S. 2007, A\&A, 468, 721

Bonfils, X., Delfosse, X., Udry, S., et al. 2013, A\&A, 549, A109

Borucki, W. J., Koch, D. G., Basri, G., et al. 2011, ApJ, 736, 19

Brown, T. M., Latham, D. W., Everett, M. E., \& Esquerdo, G. A. 2011, AJ, 142,112

Burke, C. J., Bryson, S. T., Mullally, F., et al. 2014, ApJS, 210, 19

Cochran, W. D., Hatzes, A. P., Butler, R. P., \& Marcy, G. W. 1997, ApJ, 483, 457

Cumming, A., Butler, R. P., Marcy, G. W., et al. 2008, PASP, 120, 531

Daemgen, S., Hormuth, F., Brandner, W., et al. 2009, A\&A, 498, 567

Demarque, P., Woo, J.-H., Kim, Y.-C., \& Yi, S. K. 2004, ApJS, 155, 667

Desidera, S., \& Barbieri, M. 2007, A\&A, 462, 345

Doyle, L. R., Carter, J. A., Fabrycky, D. C., et al. 2011, Sci, 333, 1602

Dressing, C. D., Adams, E. R., Dupree, A. K., Kulesa, C., \& McCarthy, D. 2014, arXiv: 1407.1848

Dressing, C. D., \& Charbonneau, D. 2013, ApJ, 767, 95

Duchêne, G., \& Kraus, A. 2013, ARA\&A, 51, 269

Duquennoy, A., \& Mayor, M. 1991, A\&A, 248, 485

Dvorak, R. 1982, OAWMN, 191, 423

Eggenberger, A., \& Udry, S. 2007, arXiv:0705.3173

Eggenberger, A., Udry, S., Chauvin, G., et al. 2011, in IAU Symp. 276, The Astrophysics of Planetary Systems: Formation, Structure, and Dynamical Evolution, ed. A. Sozzetti, M. G. Lattanzi, \& A. P. Boss (Cambridge: Cambridge Univ. Press), 409

Eggenberger, A., Udry, S., \& Mayor, M. 2004, A\&A, 417, 353

Feiden, G. A., \& Chaboyer, B. 2012, ApJ, 757, 42

Fischer, D. A., \& Marcy, G. W. 1992, ApJ, 396, 178

Fressin, F., Torres, G., Charbonneau, D., et al. 2013, ApJ, 766, 81

Gaidos, E. 2013, ApJ, 770, 90

Gaidos, E., \& Mann, A. W. 2013, ApJ, 762, 41

Giguere, M. J., Fischer, D. A., Howard, A. W., et al. 2012, ApJ, 744, 4

Gilliland, R. L., Star, K. M., Adams, E. R., et al. 2014, arXiv:1407.1009 
Ginski, C., Mugrauer, M., Seeliger, M., \& Eisenbeiss, T. 2012, MNRAS, 421 2498

Hale, A. 1994, AJ, 107, 306

Hayward, T. L., Brandl, B., Pirger, B., et al. 2001, PASP, 113, 105

Horch, E. P., Howell, S. B., Everett, M. E., \& Ciardi, D. R. 2012, AJ, 144, 165

Horch, E. P., Veillette, D. R., Baena Gallé, R., et al. 2009, AJ, 137, 5057

Howard, A. W., Marcy, G. W., Johnson, J. A., et al. 2010, Sci, 330, 653

Huber, D., Silva Aguirre, V., Matthews, J. M., et al. 2014, ApJS, 211, 2

Jang-Condell, H. 2007, ApJ, 654, 641

Jenkins, J. M., Caldwell, D. A., Chandrasekaran, H., et al. 2010a, ApJL, 713, L87

Jenkins, J. M., Chandrasekaran, H., McCauliff, S. D., et al. 2010b, Proc. SPIE, 7740,0

Kane, S. R., Howell, S. B., Horch, E. P., et al. 2014, ApJ, 785, 93

Kley, W., \& Nelson, R. P. 2008, A\&A, 486, 617

Knutson, H. A., Fulton, B. J., Montet, B. T., et al. 2014, ApJ, 785, 126

Konacki, M. 2005, ApJ, 626, 431

Konacki, M., Muterspaugh, M. W., Kulkarni, S. R., \& Hełminiak, K. G. 2009, АpJ, 704, 513

Kopparapu, R. K. 2013, ApJL, 767, L8

Kraus, A. L., \& Hillenbrand, L. A. 2007, AJ, 134, 2340

Kraus, A. L., Ireland, M. J., Hillenbrand, L. A., \& Martinache, F. 2012, ApJ, 745,19

Law, N. M., et al. 2013, arXiv:1312.4958

Lillo-Box, J., Barrado, D., \& Bouy, H. 2012, A\&A, 546, A10

Lillo-Box, J., Barrado, D., \& Bouy, H. 2014, A\&A, 566, A103

Lissauer, J. J., Ragozzine, D., Fabrycky, D. C., et al. 2011, ApJS, 197, 8

Lloyd, J. P., Liu, M. C., Macintosh, B. A., et al. 2000, Proc. SPIE, 4008, 814

Luhman, K. L., \& Jayawardhana, R. 2002, ApJ, 566, 1132

Mann, A. W., Gaidos, E., Lépine, S., \& Hilton, E. J. 2012, ApJ, 753, 90
Marcy, G. W., Isaacson, H., Howard, A. W., et al. 2014, ApJS, 210, 20

Mayor, M., et al. 2011, arXiv:1109.2497

Mugrauer, M., \& Neuhäuser, R. 2009, A\&A, 494, 373

Mugrauer, M., Neuhäuser, R., \& Mazeh, T. 2007, A\&A, 469, 755

Paardekooper, S.-J., Thébault, P., \& Mellema, G. 2008, MNRAS, 386, 973

Parker, R. J., \& Quanz, S. P. 2013, MNRAS, 436, 650

Patience, J., White, R. J., Ghez, A. M., et al. 2002, ApJ, 581, 654

Petigura, E. A., Howard, A. W., \& Marcy, G. W. 2013, PNAS, 110, 19273

Petigura, E. A., Marcy, G. W., \& Howard, A. W. 2013, ApJ, 770, 69

Quintana, E. V., Adams, F. C., Lissauer, J. J., \& Chambers, J. E. 2007, ApJ, 660,807

Raghavan, D., Henry, T. J., Mason, B. D., et al. 2006, ApJ, 646, 523

Raghavan, D., McAlister, H. A., Henry, T. J., et al. 2010, ApJS, 190, 1

Roell, T., Neuhäuser, R., Seifahrt, A., \& Mugrauer, M. 2012, A\&A, 542, A92

Sarlot, R. J., McCarthy, D. W., Burge, J. H., \& Ge, J. 1999, Proc. SPIE, 3779, 274

Schwamb, M. E., Orosz, J. A., Carter, J. A., et al. 2013, ApJ, 768, 127

Smith, J. A., Tucker, D. L., Kent, S., et al. 2002, AJ, 123, 2121

Swift, J. J., Johnson, J. A., Morton, T. D., et al. 2013, ApJ, 764, 105

Thébault, P. 2011, CeMDA, 111, 29

Thébault, P., Marzari, F., \& Scholl, H. 2006, Icar, 183, 193

Toyota, E., Itoh, Y., Ishiguma, S., et al. 2009, PASJ, 61, 19

Vogt, S. S., Allen, S. L., Bigelow, B. C., et al. 1994, Proc. SPIE, 2198, 362

Wang, J., Xie, J.-W., Barclay, T., \& Fischer, D. A. 2014, ApJ, 783, 4

Welsh, W. F., Orosz, J. A., Carter, J. A., et al. 2012, Natur, 481, 475

Wizinowich, P. L., Acton, D. S., Lai, O., et al. 2000, Proc. SPIE, 4007, 2

Wright, J. T., Marcy, G. W., Howard, A. W., et al. 2012, ApJ, 753, 160

Xie, J.-W., Zhou, J.-L., \& Ge, J. 2010, ApJ, 708, 1566 